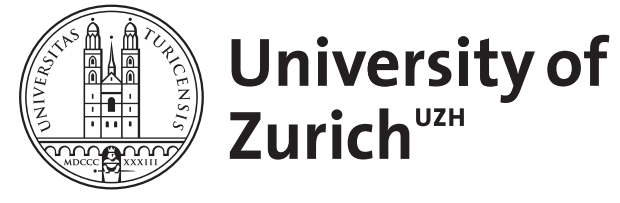

\title{
Intraossäre Infusionstechnik
}

Weiss, M ; Gächter-Angehrn, J ; Neuhaus, D

\begin{abstract}
Die intraossäre Infusionstechnik ist zum festen Bestandteil in der pädiatrischen aber auch adulten Notfallmedizin geworden und gehört heute zu den wichtigsten Fertigkeiten eines Notarztes, Pädiaters, Anästhesisten und Intensivmediziners. Die intraossäre Infusionstechnik ist eine sichere, zuverlässige und schnelle Methode, um bei Kindern unter 6Jahren mit Kreislaufstillstand oder schwerem hypovolämischem Schock primär und beim vital gefährdeten Kind mit schwierigen Venenverhältnissen sekundär einen Gefäßzugang zu legen. Auf diesem Weg können alle in der Notfallmedizin intravenös verabreichten Medikamente und Flüssigkeiten nahezu uneingeschränkt, im Vergleich zur intravenösen Gabe in äquivalenter Dosierung und mit vergleichbaren Anschlagszeiten appliziert werden. Kenntnisse über Indikationen, Punktionsstellen, Material, Technik, Vorsichtsmaßnahmen und Komplikationen sowie Training am Übungsknochen sind wichtig für den sicheren und erfolgreichen klinischen Einsatz. Bei richtiger Indikationsstellung gibt es abgesehen von lokalen Gründen wie Frakturen, voroperierter Knochen oder kürzlich vorausgegangene Punktionsversuche sowie Durchblutungsstörungen der betroffenen Extremität keine absoluten Kontraindikationen für eine intraossäre Infusion. Komplikationen sind bei richtiger Anwendung und Handhabung extrem selten und das Risiko steht in keinem Verhältnis zu den Vorteilen einer intraossären Infusion beim vital bedrohten Patienten
\end{abstract}

DOI: https://doi.org/10.1007/s10049-007-0900-0

Posted at the Zurich Open Repository and Archive, University of Zurich

ZORA URL: https://doi.org/10.5167/uzh-156417

Journal Article

Published Version

Originally published at:

Weiss, M; Gächter-Angehrn, J; Neuhaus, D (2007). Intraossäre Infusionstechnik. Notfall Rettungsmedizin, 10(2):99-116.

DOI: https://doi.org/10.1007/s10049-007-0900-0 
Notfall Rettungsmed 2007 · 10:99-116

DOI 10.1007/s10049-007-0900-0

Online publiziert: 8. März 2007

(c) Springer Medizin Verlag 2007

\section{Redaktion}

T. Nicolai, München
M. Weiss · J. Gächter-Angehrn · D. Neuhaus

Anästhesieabteilung, Universitäts-Kinderkliniken Zürich

Infusionstechnik

\section{(3) plus Video}

Das Anlegen eines Gefäßzugangs beim kritisch kranken oder verletzten Säugling und Kleinkind ist auch in den Händen von erfahrenem Medizinalpersonal nicht immer innerhalb nützlicher Frist gewährleistet [1]. Säuglinge und Kleinkinder haben bereits von vornherein durch die kleineren anatomischen Verhältnisse, wie die dickere subkutane Schicht, schwierigere Punktionsverhältnisse. Kommen zusätzlich Hypovolämie, Vasokonstriktion und/oder Kälte dazu, wird es auch für den Erfahrenen sehr schwierig oder gar unmöglich, einen Venenzugang anzulegen.

Die intraossäre Infusionstechnik hat sich in den letzten 20 Jahren als sichere Alternativtechnik zur schnellen Etablierung eines Gefäßzugangs bei dringend benötigter Medikamenten- und Flüssigkeitsgabe bei vitalgefährdeten Kindern entwickelt (- Abb. 1). Sie ist mittlerweile auch als fester Bestandteil in den Reanimations- und Notfallmedizinrichtlinien des European Resuscitation Council (ERC), der American Heart Association (AHA) und des International Liason Committee of Resuscitation (ILCOR) für Kindern und Erwachsene verankert [2, 3, 4].

Die intraossäre Infusion bietet vor allem dem in der pädiatrischen Notfallund Rettungsmedizin wenig erfahrenen Arzt oder Rettungssanitäter die Möglichkeit, schnell, effizient und sicher einen Gefäßzugang zur dringend benötigten $\mathrm{Me}$ dikamenten- und Flüssigkeitsgabe beim vital gefährdeten Kind zu etablieren.

Obwohl heute die intraossäre Infusionstechnik einen international anerkannten alternativen Gefäßzugang in der Notfallmedizin darstellt, ist die intraossäre Infusionstechnik oft noch ein „Tabu" und wird entsprechend auch zu we- nig eingesetzt. Mangel an Kenntnissen, fehlende Übung sowie Hemmungen verhindern oft die Anwendung dieser lebensrettenden Technik. Die folgende Arbeit soll eine Übersicht über die Geschichte, Methodik sowie Indikationen und Risiken der intraossären Infusionstechnik hauptsächlich im Hinblick auf den pädiatrischen Notfallpatienten geben.

\section{Historisches}

Anfang der 1920er Jahre wurde erstmals auf die Möglichkeit der Verabreichung von Flüssigkeiten durch einen intraossären Zugang hingewiesen $[5,6,7]$. In den 1940er Jahren förderte Tocantins die Einführung der intraossären Infusionstechnik in die klinische Praxis $[8,9,10,11,12$, 13, 14]. Durch Verbesserung von Ausrüstung und Technik entwickelte sie sich bald $\mathrm{zu}$ einer oft benutzten Methode bei Kindern für die parenterale Gabe von Flüssigkeiten, Blut und Medikamenten über mehrere Tage [14]. Im 2. Weltkrieg erlangte die intraossäre Infusion als Zugang für die Volumentherapie ihre Bedeutung auch bei erwachsenen Patienten [15]. Als in den 1950er Jahren Plastikkanülen zur intravenösen Katheterisierung aufkamen, geriet die intraossäre Infusionstechnik weitgehend in Vergessenheit und wurde sogar als Obskurität angesehen.

1983 veröffentlichte Turkel einen Brief im "American Journal of Diseases of Children", in dem er fragte, warum die intraossäre Technik in pädiatrischen Notfallsituationen ignoriert werde, obwohl sie in vielen Situationen lebensrettend sein könnte. Anlass zu diesem Brief war der Tod eines Kindes mit Tonsillennachblutung nach vergeblicher Suche eines intravenösen Zugangs zur Anästhesie- induktion [16]. Diese und weitere Publikationen waren Anfang der 1980er Jahre ausschlaggebend, dass die intraossäre Infusionstechnik eine Renaissance als $\mathrm{Ge}$ fäßzugang in Notfallsituationen bei $\mathrm{Pa}$ tienten mit schwierigen oder fehlenden Venenverhältnissen erlebte und heute als solcher in der pädiatrischen Notfalltherapie fest verankert ist $[2,3,4,17]$.

\section{Prinzip der intraossären Infusion}

Die intraossäre Infusion ist im Grunde genommen eine Infusion in den intramedullären Blutgefäßraum des roten Knochenmarks. Dabei können mittels einer durch den Knochenkortex in die Markhöhle eingeführten Nadel Infusionslösungen, Blutprodukte und Medikamente ins Gefäßsystem verabreicht werden. Diese gelangen direkt in das Netzwerk der venösen Marksinusoide, von dort in den Zentralvenenkanal des Knochenmarks und danach via Vv. nutrientes und Vv. emissariae in den venösen Systemkreislauf (• Abb. 2). Die intramedullären Gefäße und Sinusoide im Knochenmark sind von harten Knochenwänden gestützt und geschützt, die auch bei Schock und Herzstillstand nicht kollabieren.

Bei der Geburt sind die Markhöhlen aller Knochen mit hämatopoetischen Zellen gefüllt, die als rotes Knochenmark bezeichnet werden. Im Verlauf der Entwicklung des Kindes ersetzen Fettzellen (gelbes Knochenmark) zunehmend das blutbildende rote Knochenmark, sodass rotes Knochenmark später nur noch in Wirbelkörpern, Rippen, Schädel, Sternum, Becken und in den Metaphysen der Röhrenknochen gefunden wird. Letztere stellen die Hauptpunktionsorte in der heutigen klinischen Praxis bei Kindern dar. 


\section{Leitthema}

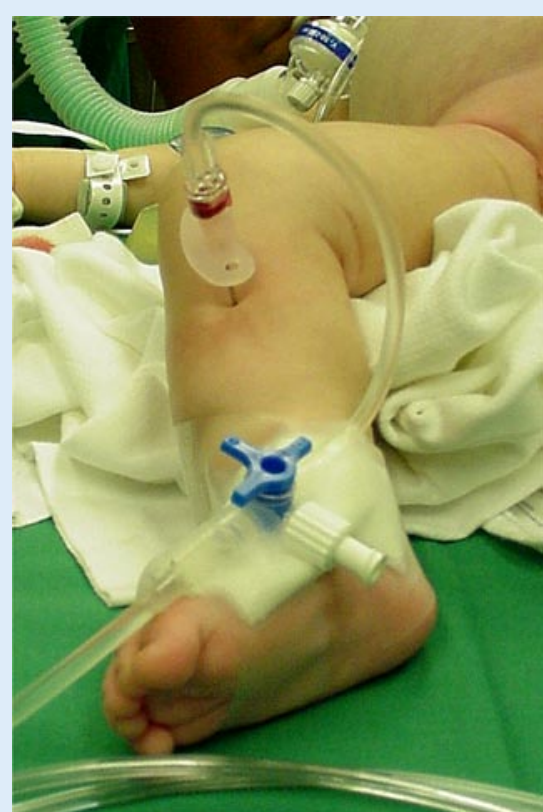

Abb. 1 A Intraossärnadel in der rechten Tibia eines 11 Monate alten Säuglings

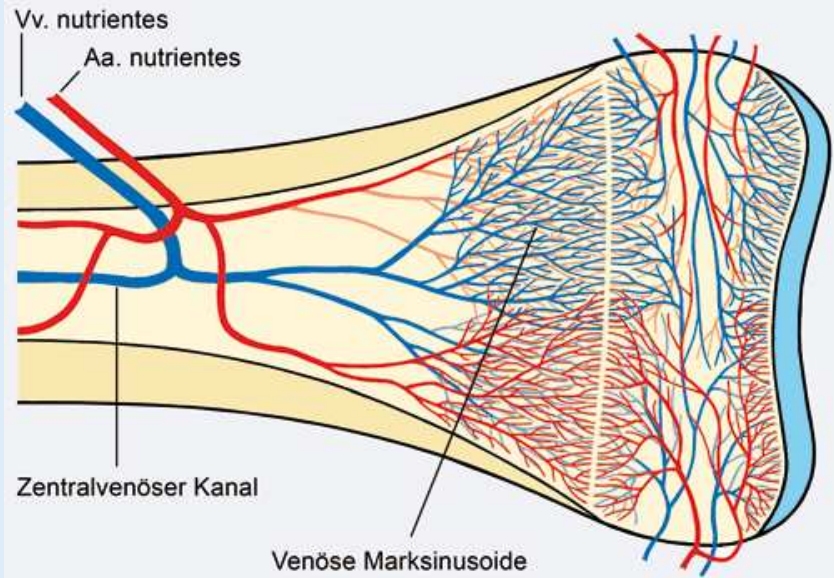

Abb. $2 \triangle$ Prinzip der intramedullären Zirkulation

Abb. $3>$ Punktionsstelle an der proximalen

Tibia

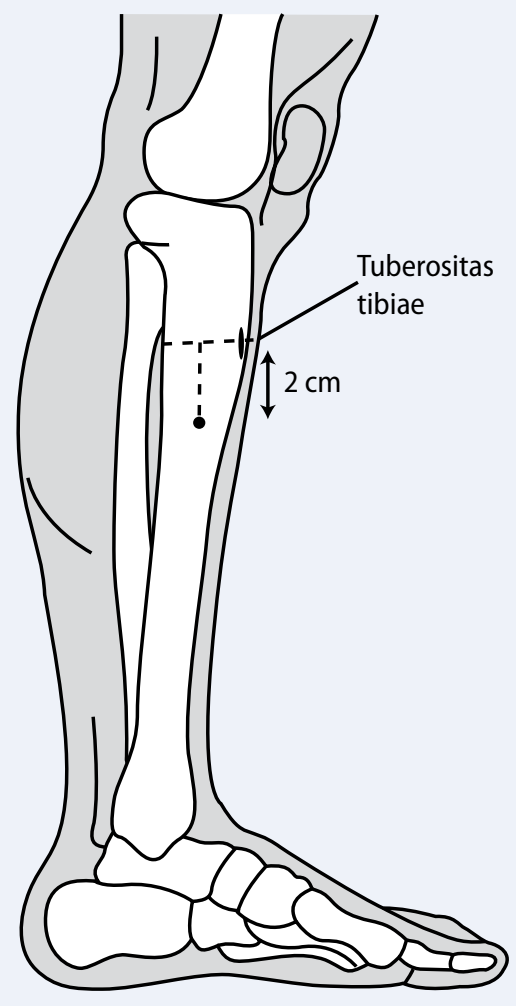

Die Punktion des Knochens ist vergleichbar mit der Punktion einer knöchernen Vene, die auch bei Kälte, Vasokonstriktion und Hypovolämie nicht kollabiert.

\section{Punktionsstellen}

In den 1940er Jahren war das Sternum der übliche intraossäre Punktionsort bei erwachsenen Patienten $[8,18]$, da diese Punktionsstelle gut zugänglich und markhaltig ist. Nur wenige Autoren empfahlen diese Punktionsstelle auch bei Kindern [19]. Aufgrund letaler Komplikationen und der Unvereinbarkeit mit extrathorakaler Herzkompression wurde dieser Punktionsort weitgehend verlassen. Vor allem bei Kindern sollte auf keinen Fall eine Punktion des Sternums durchgeführt werden $[9,10]$, da das Risiko einer Punktion des Mediastinums und somit eine Verletzung des Herzens oder der großen Gefäße erheblich ist [20]. Das Knochenmark im Sternum von Kindern ist unregelmäßig verteilt und nicht groß genug, um die transfundierten Flüssigkeitsmengen adäquat aufzunehmen [11, 21]. Außerdem ist das Sternum beim Kind zu dünn und un- terentwickelt, um eine sichere, stabile Platzierung der Nadel zu gewährleisten.

Charakteristika einer geeigneten Punktionsstelle für die IOT sind in $\bullet$ Tab. 1 aufgeführt. Die proximale Tibia stellt die optimale Stelle für die intraossäre Infusion bei Kindern unter 6 Jahren in Notfallsituationen dar [22, 23]. Des Weiteren kommt die Punktion an der distalen Tibia und am distalen Femur in Frage. Alternativ kann bei großen Becken- und Abdominaltraumen mit ungewissem Blutfluss in den Thorax der proximale Humerus punktiert werden. 
Indikationen für die Intraossärnadel

beim Kind $<6$ Lebensjahren

- Primär: Herzstillstand oder ausgeprägter hypovolämer Schock

- Sekundär: vital bedrohte Kinder, falls nach $90-120$ s intravenöse Punktionsversuche erfolglos blieben

\section{Punktion an der proximalen Tibia}

Der Punktionsort befindet sich auf der flachen, breiten anteromedialen Oberfläche der proximalen Tibia, ca. $1-2 \mathrm{~cm}$ (1-2 Fingerbreiten) unterhalb der palpablen Tuberositas tibiae ( $\bullet$ Abb. 3). Die Tibia soll einerseits wegen der Verletzungsgefahr der Wachstumsfuge beziehungsweise des Kniegelenks nicht zu proximal eingestochen werden. Andererseits sollte sie auch nicht zu distal punktiert werden, da der Knochen dichter und daher schwieriger zu punktieren ist. Wichtig ist, darauf zu achten, dass nicht auf der Tibiavorderkante punktiert wird, da hier der Knochen sehr hart ist, was zu Fehlpunktionen und Komplikationen führt. Die Nadel wird in einem Winkel von $90^{\circ}$ zur Haut- bzw. Knochenoberfläche angesetzt.

Die intraossäre Punktion in die proximale Tibia ist beim ersten Versuch in $77 \%$ der Fälle erfolgreich und benötigt in den meisten Fällen weniger als 1 min [24]. Diese Erfolgsquote basiert auf den zahlreichen Vorteilen dieser Punktionsstelle. Die proximale Tibia sollte mit den manuellen Handnadeln nur bis zum Alter von 5-6 Jahren punktiert werden. Danach wird diese Stelle zu dick und ist zunehmend schwieriger zu durchbohren [22].

Im Gegensatz zum Kind, bei welchem sich die intraossäre Punktionsstelle an der proximalen Tibia 1-2 $\mathrm{cm}$ unterhalb der Tuberositas befindet, wird beim Erwachsenen auf Höhe der Tuberositas (EZ- $\mathrm{IO}^{\mathrm{Tw}}$ Intraossärinfusionssystem) oder $1 \mathrm{~cm}$ darüber (Bone Injection Gun, BIG) punktiert. Dies nicht zuletzt, weil der dünnere Knochenkortex im Bereich gegen die Epiphyse einfacher zu durchdringen ist als die dicke Kompakta im diaphysären Bereich.

\section{Punktion an der distalen Tibia}

Bei der Punktion der distalen Tibia liegt die optimale Einstichstelle in der kranialen Verlängerung der Mittellinie des medialen

Notfall Rettungsmed 2007 $\cdot 10: 99-116 \quad$ DOI 10.1007/s10049-007-0900-0

(c) Springer Medizin Verlag 2007

M. Weiss · J. Gächter-Angehrn · D. Neuhaus Intraossäre Infusionstechnik

\section{Zusammenfassung}

Die intraossäre Infusionstechnik ist zum festen Bestandteil in der pädiatrischen aber auch adulten Notfallmedizin geworden und gehört heute zu den wichtigsten Fertigkeiten eines Notarztes, Pädiaters, Anästhesisten und Intensivmediziners. Die intraossäre Infusionstechnik ist eine sichere, zuverlässige und schnelle Methode, um bei Kindern unter 6 Jahren mit Kreislaufstillstand oder schwerem hypovolämischem Schock primär und beim vital gefährdeten Kind mit schwierigen Venenverhältnissen sekundär einen Gefäßzugang zu legen. Auf diesem Weg können alle in der Notfallmedizin intravenös verabreichten Medikamente und Flüssigkeiten nahezu uneingeschränkt, im Vergleich zur intravenösen Gabe in äquivalenter Dosierung und mit vergleichbaren Anschlagszeiten appliziert werden. Kenntnisse über Indikationen, Punk-

\section{Technique of intraosseous infusion}

\section{Abstract}

Intraosseous infusion has become a cornerstone in pediatric and adult emergency medicine and belongs to one of the most important knowledge and skills of an emergency physician, pediatrician, anesthetist and intensivists. Intraosseous infusion is a safe, reliable and fast technique for primary vascular access in children $<6$ years of age with cardiac arrest or severe hypovolaemic shock and for secondary vascular access in the child in lifethreatening conditions and difficult venous access. All drugs and fluids used in emergency medicine can be used by the intraosseous route almost without restriction and in similar dosage and efficiency as by the venous route. Besides local factors such as fracture, previous orthopedic or surgical procedures, recent intraosseous puncture as well as vas- tionsstellen, Material, Technik, Vorsichtsmaßnahmen und Komplikationen sowie Training am Übungsknochen sind wichtig für den sicheren und erfolgreichen klinischen Einsatz. Bei richtiger Indikationsstellung gibt es abgesehen von lokalen Gründen wie Frakturen, voroperierter Knochen oder kürzlich vorausgegangene Punktionsversuche sowie Durchblutungsstörungen der betroffenen Extremität keine absoluten Kontraindikationen für eine intraossäre Infusion. Komplikationen sind bei richtiger Anwendung und Handhabung extrem selten und das Risiko steht in keinem Verhältnis zu den Vorteilen einer intraossären Infusion beim vital bedrohten Patienten.

\section{Schlüsselwörter}

Intraossäre Infusion · Gefäßzugang ·

Punktion · Knochenmark

cular disturbances of the extremity there are no general contraindications for intraosseous infusion if indication is chosen correctly. Incidence of complications is low if intraosseous infusion technique is correctly performed and the potential risks are negligible compared with the advantages for the life-threatened child. Knowledge about indications, sites of puncture, equipment, technique, precautions and complications as well as training with mannequin bones are mandatory for the safe and successful clinical use of this technique.

Keywords Intraosseous infusion - Vascular access . Punction · Bone marrow 
Tab. 1 Kriterien für geeignete Punktionsstellen zur intraossären Infusion

Oberflächliche Lage des zu punktierenden Knochens

Leicht auffindbare Orientierungspunkte oder Landmarken

Punktionsort ist gut zugänglich und erlaubt auch Manipulationen am Patienten in Rückenlage (Intubation, Herzmassage)

Flache Knochenoberfläche zur Vermeidung des Abrutschens bei der Punktion

Dünne Knochenkompakta zur Erleichterung der Penetration der Nadel

Geräumige Markhöhle zur leichteren Infusion Rotes Knochenmark im Punktionsbereich zur besseren Aufnahme des Injektats in den Kreislauf

Keine Nerven, Gefäße oder sonstige verletzlichen Strukturen vor oder hinter dem punktierten Knochen

Tab. 2 Material für das Anlegen und

Betreiben sowie zur Sicherung und Fixation einer intraossären Infusion

Sterile Handschuhe

Desinfektionsmittel

Sterile Tupfer zur Desinfektion

Intraossärnadeln

Lochtuch (optional)

Lokalanästhetikum (inkl. Spritze und Nadel)

Infusionsleitung mit Dreiwegehahn und Verlängerung

Klebeband

Lagerungsschiene, elastische Binde

Malleolus, $2-3 \mathrm{~cm}$ oberhalb der malleolären Kuppe (• Abb.4). An dieser Stelle können keine Strukturen beschädigt werden, insbesondere nicht die V. saphena und die Wachstumsfuge. Die Nadel wird in einem Winkel von $90^{\circ}$ zur Haut- bzw. Knochenoberfläche angesetzt. Diese Stelle ist günstig für eine intraossäre Injektion, da der Knochen oberflächlich liegt und relativ flach ist.

Die distale Tibia sollte zur intraossären Punktion verwendet werden, falls an der proximalen Tibia keine Punktion möglich ist (Fraktur, vorausgehende Fehlpunktion). Im Gegensatz zur proximalen Tibia kann beim Einsatz von manuellen Intraossärnadeln die distale mediale Tibia als Punktionsort auch bei älteren Kindern und Erwachsenen verwendet werden und ist daher die bevorzugte Punktionsstelle für intraossäre Nadeln bei Kindern $>6$ Lebensjahren und bei Erwachsenen [25, 26].
Von einigen Autoren wird diese Punktionsstelle gegenüber der proximalen Tibia sogar generell bevorzugt [25].

\section{Punktion am distalen Femur}

Die Punktionsstelle am distalen Femur, an der Mittellinie der Vorderfläche des Femurs, $1-2 \mathrm{~cm}$ oberhalb der Patella $(2-3 \mathrm{~cm}$ oberhalb der Kondylen). Die Nadel wird in einem Winkel von $90^{\circ}$ zur Femuroberfläche angesetzt. An dieser Stelle können nur wenige Strukturen verletzt werden. Das darunter befindliche Knochenmark ist relativ groß [23], sodass höhere Flussraten erreicht werden können [27].

Beim distalen Femur handelt es sich normalerweise nicht um den Punktionsort der ersten Wahl. Es ist schwieriger, durch die Weichteile hindurch die knöcherne Punktionsstelle zu finden. Der Kortex ist dichter und schwieriger zu penetrieren. Die Nadel kann leichter abrutschen und dadurch die Nachbarstrukturen verletzen [28]. Zusätzlich muss durch eine dicke Muskel- und Fettschicht punktiert werden, weshalb eine längere intraossäre Nadel benötigt wird [29]. Damit sind Tiefenmarkierungen auf Intraossärnadeln nicht adäquat und gewisse Nadeltypen gar zu kurz. Weiterer Nachteil ist, dass durch die Sehne des M. quadriceps punktiert wird, sodass bei Flexion oder Extension dieses Muskels die intraossäre Nadel dislozieren kann [30].

\section{Punktion am proximalen Humerus}

Der Humerus wird als Punktionsort zur intraossären Infusion bei Erwachsenen in der Literatur aufgeführt [31], hat aber nie an Beliebtheit gewonnen. Nach gröBeren Verletzungen des Beckens oder Abdomens mit beeinträchtigem venösem Rückfluss, mag eine intraossäre Infusion oberhalb des Diaphragmas sinnvoll sein. In diesen Situationen bietet sich der proximale Humerus als intraossäre Punktionsstelle an [32]. In neuester Zeit gewinnt dieser Zugang in der Erwachsenenmedizin an Akzeptanz, da bei engen Platzverhältnissen, z. B. in einer Ambulanz, die intraossäre Infusion auch von einem am Kopf des Patienten stehenden oder sitzenden Helfer angelegt werden kann. Dieser $\mathrm{Zu}$ gang ist seit kurzem auch vom Hersteller für das EI-IO ${ }^{\text {tw }}$ Intraossärinfusionssystem beim Erwachsenen freigegeben worden.

\section{Punktion am Sternum beim Erwachsenen}

Beim Kind ist die Punktion des Sternums für die intraossäre Infusionstherapie verboten! Mit der Entwicklung von intraossären Infusionspunktionsgeräten mit limitierter Eindringtiefe wie zum Beispiel das (F.A.S.T.) wird diese Punktionsstelle jedoch in neuster Zeit bei erwachsenen Patienten wieder propagiert [26,31, 33]. Vorteil des Sternums zur intraossären Punktion beim Erwachsenen sind die dünne Kompakta, welche eine einfache Penetration erlaubt, und die gute Flüssigkeitsaufnahme, aufgrund der reichlichen venösen Drainage des Sternums unter dem Einfluss des negativen thorakalen Venendrucks. Letzteres erlaubt, größere Flüssigkeitsmengen pro Zeit beim erwachsenen Patienten zu infundieren.

\section{> Beim Kind ist die Punktion des Sternums zur intraossären Infusion verboten!}

\section{Punktionsmaterial}

Seit der Entdeckung der intraossären Infusionstechnik in den 1920er und 1930er Jahren sind eine Vielzahl an Punktionskanülen (• Abb. 5) und Apparaten/Vorrichtungen (• Abb. 6) für die intraossäre Infusion vorgeschlagen, entwickelt und in die Praxis eingeführt worden. Grundsätzlich unterscheidet man zwischen Nadeln und Apparaten/Vorrichtungen zum Anlegen einer intraossären Infusion.

\section{Punktionsnadeln}

Im Prinzip kann jede Stahlkanüle, die ein inneres Stilett hat und somit beim Einführen nicht verstopft, für die intraossäre Infusion verwendet werden (• Abb. 7). Eine ideale Intraossärnadel zeichnet sich aus durch:

- einen möglichst großkalibrigen Schaft zur erleichterten Infusion,

- eine besonders geschliffene Spitze

(Trokar) zur besseren Knochenpenetration, 


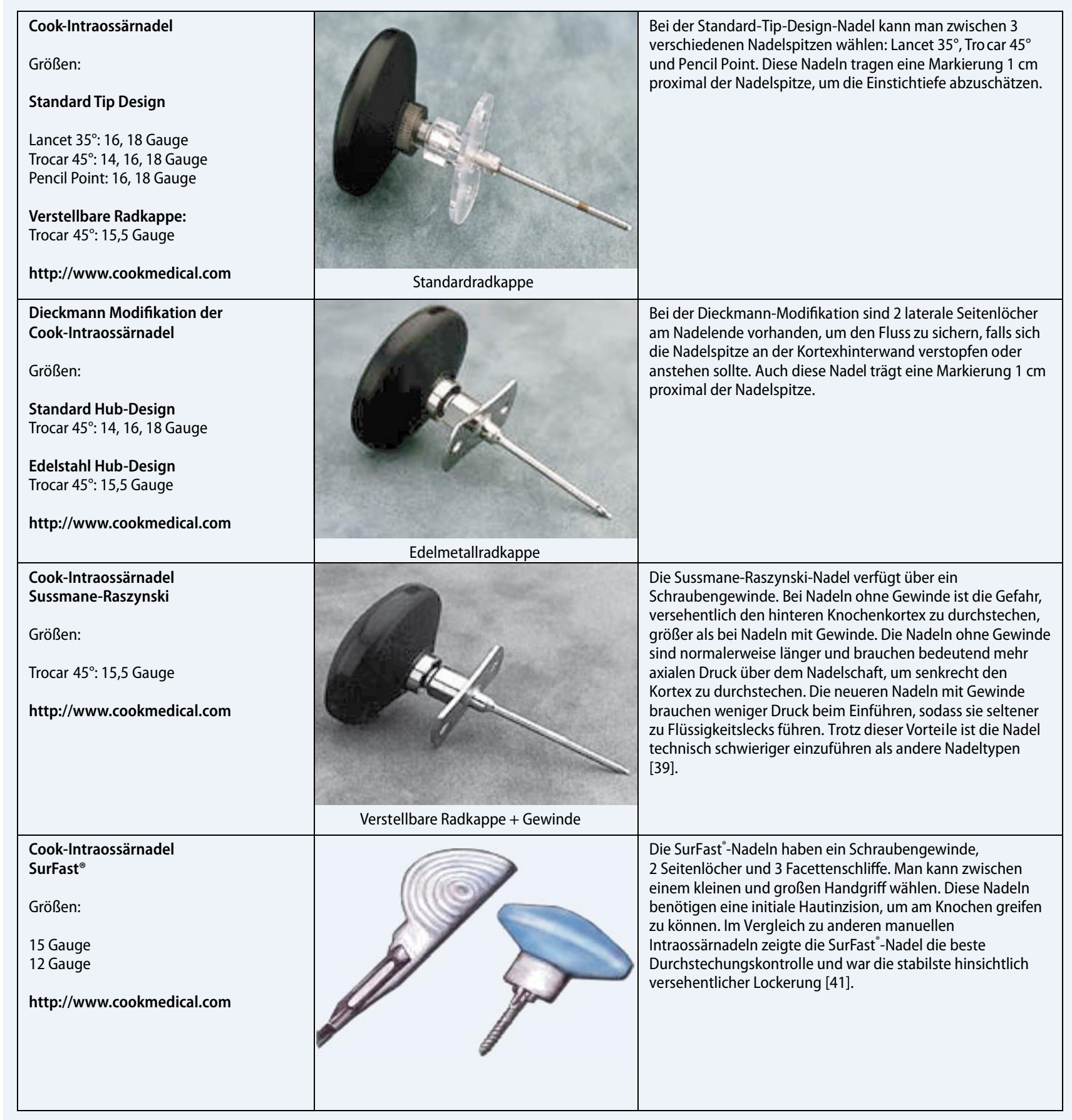

Abb. $5 \Delta$ Kommerziell erhältliche Nadeln für die intraossäre Infusion

- einen kurzen, soliden Schaft für eine gute Längs- und Drehstabilität,

- einen Handgriff zur Kraftübertragung auf die Nadel,

- einen Trokar zur Vermeidung der Lumenverlegung bei der Punktion,

- seitliche Öffnungen zum Offenhalten der Nadel bei Kontakt mit dem gegenüberliegenden Endost oder gar Penetration in den Kortex,
- die Möglichkeit, die Eindringtiefe abzulesen oder gar die maximale Eindringtiefe einzustellen

- einen Anschluss für Luer-Lock-Standardinfusionssets.

Ein Nachteil bei der Benutzung einer nicht speziell für die intraossäre Infusion konzipierten Nadel ist, dass die Zeit für das Setzen der Nadel dadurch verlängert wird und die Gefahr des Verbiegens der Nadel größer ist [34, 35].

Neben kommerziell erhältlichen Intraossärnadeln (• Abb. 5) sind folgende Nadeln erfolgreich für die intraossäre Infusion angewendet worden:

- Knochenmarkbiopsie- und Aspirati-

onsnadeln (Cave: Anschlussinkompa-

tibilitäten!),

- Stahlinjektionsnadeln, 


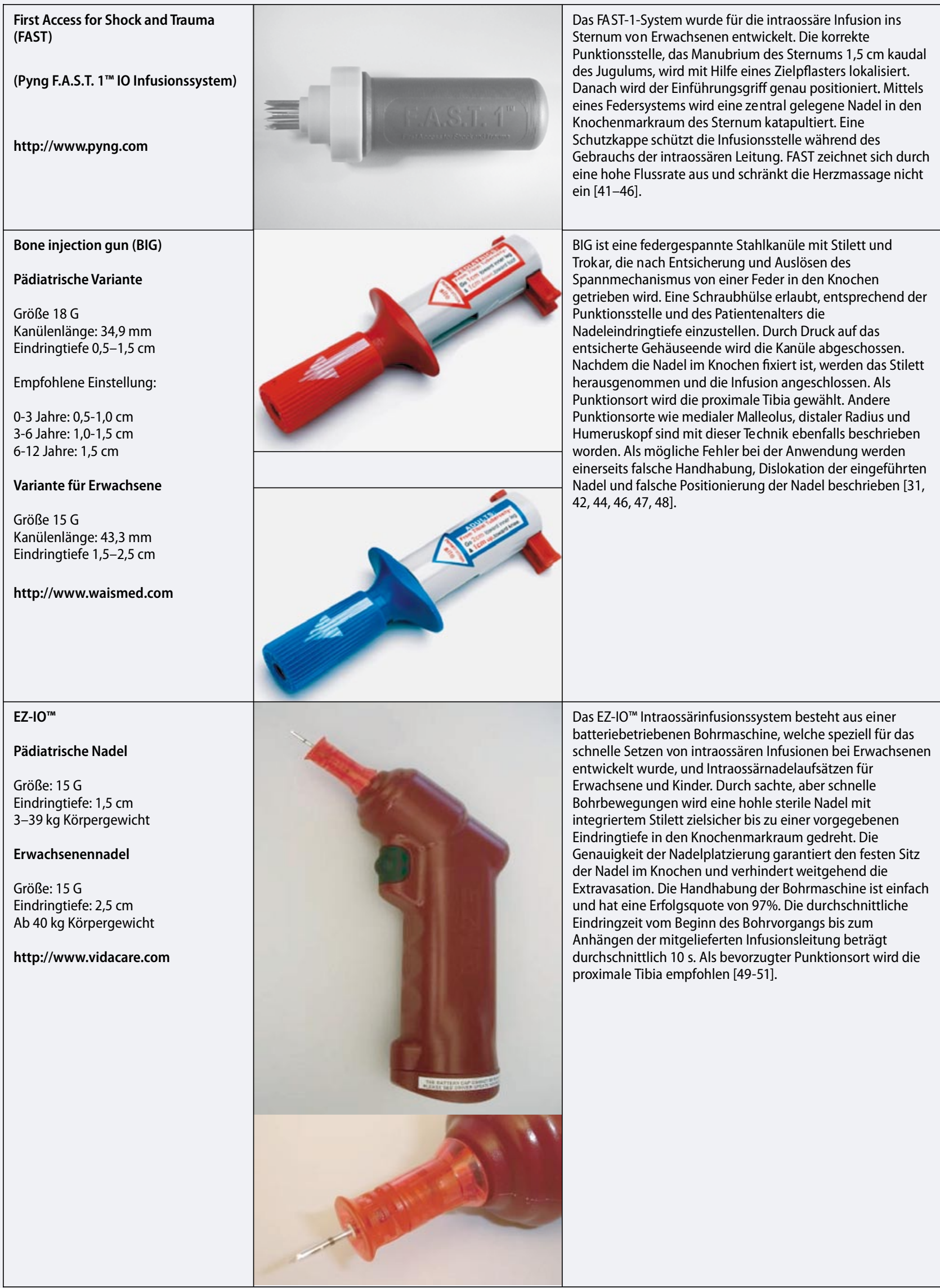

Abb. $6 \Delta$ Kommerziell erhältliche Geräte für die intraossäre Infusion 
- Spinal- bzw. Lumbalpunktionsnadeln mit Trokar (Neugeborene und Säuglinge),

- Schmetterlingsnadeln (Butterfly; [36, 37]).

Je nach Alter werden für adäquate Flussraten unterschiedliche Nadelgrößen empfohlen $[22,27,38]$ :

$<18$ Monate: $20-18 \mathrm{G}(1,1-1,3 \mathrm{~mm})$,

$>18$ Monate: $16-13 \mathrm{G}(1,8-2,5 \mathrm{~mm})$.

Im Notfall kann, falls nicht anders vorhanden, eine hohle Stahlnadel für die intraossäre Injektion verwendet werden. Plastikkanülen haben sich jedoch nicht bewährt. Bei den modernen diagnostischen Knochenmarknadeln muss darauf geachtet werden, ob der Anschluss an ein Luer-Lock-Infusionsset gewährleistet ist, ansonsten muss eine Extraverbindungsleitung dem Set beigelegt werden.

Grundsätzlich sollten aber in der Praxis die kommerziell erhältlichen Intraossärnadeln zum Einsatz kommen. In der pädiatrischen Notfallmedizin haben sich die Intraossärnadeln der Firma Cook Critical Care (Bloomington, IN, USA) bisher am besten bewährt. Dabei sind $\mathrm{Na}$ deln zu unterscheiden, die durch „Drehen und Drücken“, und Nadeln, welche durch „Schrauben“ durch den Knochenkortex ins Knochenmark eingebracht werden. Die verschiedenen Typen von Intraossärnadeln sind in $\bullet \mathbf{A b b} \mathbf{5}$ aufgelistet und kommentiert.

Der Vorteil der Intraossärnadeln mit Schraubgewinde liegt darin, dass der Kraftaufwand geringer als bei den „DrehDruck-Nadeln" ist, die Extravasation geringer und die Fixation im Knochen stabiler ist [39]. Als Nachteil jedoch fehlt der klassische Widerstandsverlust bei der Penetration in den Markraum als erstem Zeichen der erfolgreichen Punktion und einer korrekten Kanülenlage. Speziell zu erwähnen ist die Intraossärnadel mit Diekmann-Modifikation der Firma Cook Critical Care, die am distalen Ende 2 seitliche Öffnungen aufweist, welche auch bei Verlegung der Kanülenspitze (z. B. durch Anliegen der Kanülenspitze am gegenüberliegenden Endost oder Eindringen der Kanülenspitze in den gegenüberliegenden Knochenkortex) die Applikation von Flüssigkeiten und Medikamenten erlaubt.
Baxter bietet die sog. Jamshidi-Intraossärnadel mit einem verstellbaren Plastikschutz an (18G/15G). Dieser erlaubt eine bestimmte Nadellänge einzustellen, sodass die Nadel nicht zu tief eingeführt wird. Die generell kurze Nadel erlaubt eine gute Kraftübertragung. Die ganze Nadel mit Plastikschutz ist sehr handlich, was die Einführung vereinfacht [40]. Zusätzlich kann dieser Plastikschutz nach dem Einführen verstellt werden, damit sich die Nadel anschließend nicht verbiegt. Der bis auf Hautniveau gesenkte Plastikschutz erlaubt eine bessere Stabilisierung der Nadel. Die Baxter-JamshidiIntraossärnadel ist wie alle Cook-Intraossärnadeln mit Luer-Lock-Infusionssystemen kompatibel.

\section{Geräte für die intraossäre Punktion/Infusion}

Vor allem die Anwendung der intraossären Infusionstechnik bei Erwachsenen sowie in der Militärmedizin hat zur Entwicklung von Vorrichtungen geführt, welche es erlauben, auch durch harten und dicken Knochenkortex schnell und erfolgreich einen intraossären Zugang zu legen (• Abb. 6; [40]). Dabei werden Geräte mit Federmechanismus wie das Pyng F.A.S.T. ${ }^{\text {Ts }}$ IO Infusionssystem (FAST; [41, $42,43,44,45,46])$ oder die Bone Injection Gun (BIG; [31, 42, 44, 46, 48]) und solche mit Bohrmaschine (Adult EZ-IO ${ }^{\mathrm{rw}}$, Vidacare; $[49,50,51])$ unterschieden. BIG und $\mathrm{EZ}^{-\mathrm{IO}^{\mathrm{se}}}$ wurden bisher v. a. zur intraossären Infusion bei Erwachsenen angewendet und getestet. Gewisse Vorrichtungen sind aber auch für die pädiatrische Notfallmedizin sicherlich von Bedeutung, da die Kanüle schneller und technisch einfacher durch den Kortex ins Knochenmark einzubringen ist. Die Anwendung dieser Geräte wird von allen 3 Herstellern mittels Videos und Anwendungsrichtlinien auf der entsprechenden Website gezeigt (• Abb. 6).

Bei Kindern kommen heute noch vorwiegend manuelle Intraossärnadeln zum Einsatz. Automatische Federsysteme oder Bohrmaschinengeräte für die intraossäre Infusion werden vorwiegend noch bei Erwachsenen eingesetzt, haben aber in $\mathrm{Zu}$ kunft auch bei Kindern das Potenzial, die Punktionstechnik zu vereinfachen.

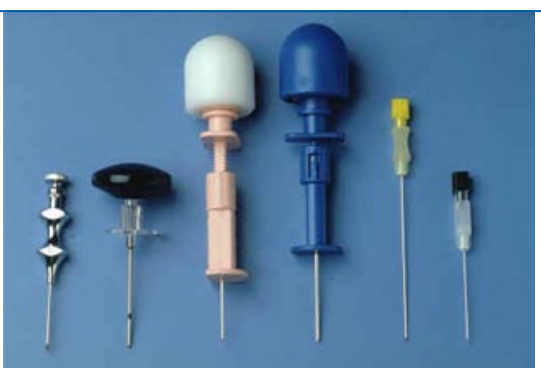

Abb. $7 \Delta$ Nadeln für die intraossäre Infusion

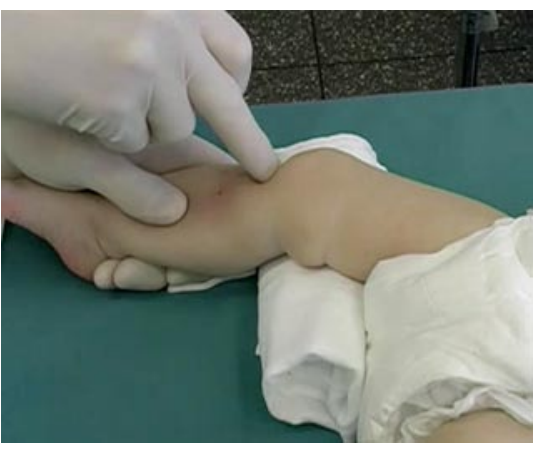

Abb. $8 \Delta$ Intraossäre Infusion bei einem 11-monatigen Säugling. Die intraossäre Punktion wurde anlässlich einer diagnostischen Knochenmarkentnahme in Allgemeinanästhesie durchgeführt. Siehe Video zum Beitrag unter www.NotfallundRettungsmedizin.de

\section{Zusätzlich benötigtes Material}

Zum Anlegen einer intraossären Infusion bedarf es je nach verwendetem Punktionsmaterial steriler Handschuhe, Desinfektionsmaterial, Fixationsmaterial, Infusionsbesteck inkl. Spritzen sowie Material zur Lokalanästhesie. Dieses Material ist zumeist in der Notarztausrüstung oder auf einer Notfallstation zu finden. Es hat sich jedoch gerade in Ambulanzen und Helikoptern bewährt, fertig abgepackte Sets für die intraossäre Infusion zusammenzustellen, um im Notfall keine Zeit mit der Materialsuche zu verlieren (• Tab. 2).

\section{Technik der intraossären Infusion}

Die Technik der intraossären Infusion (- Abb. 8) ist in $\bullet$ Abb. 9 (Cook-Intraossärnadel) und in $\bullet$ Abb. 10 (EZ-IO ${ }^{\text {Tw}}$ Intraossärinfusionssystem) Schritt für Schritt am Beispiel der proximalen Tibia, der intraossären Punktionsstelle der ersten Wahl bei Kindern unter 6 Jahren, gezeigt und erläutert.

Im Folgenden soll auf einige wichtige Punkte beim Einsatz der Cook-Intraossärnadel näher eingegangen werden: 


\section{Leitthema}

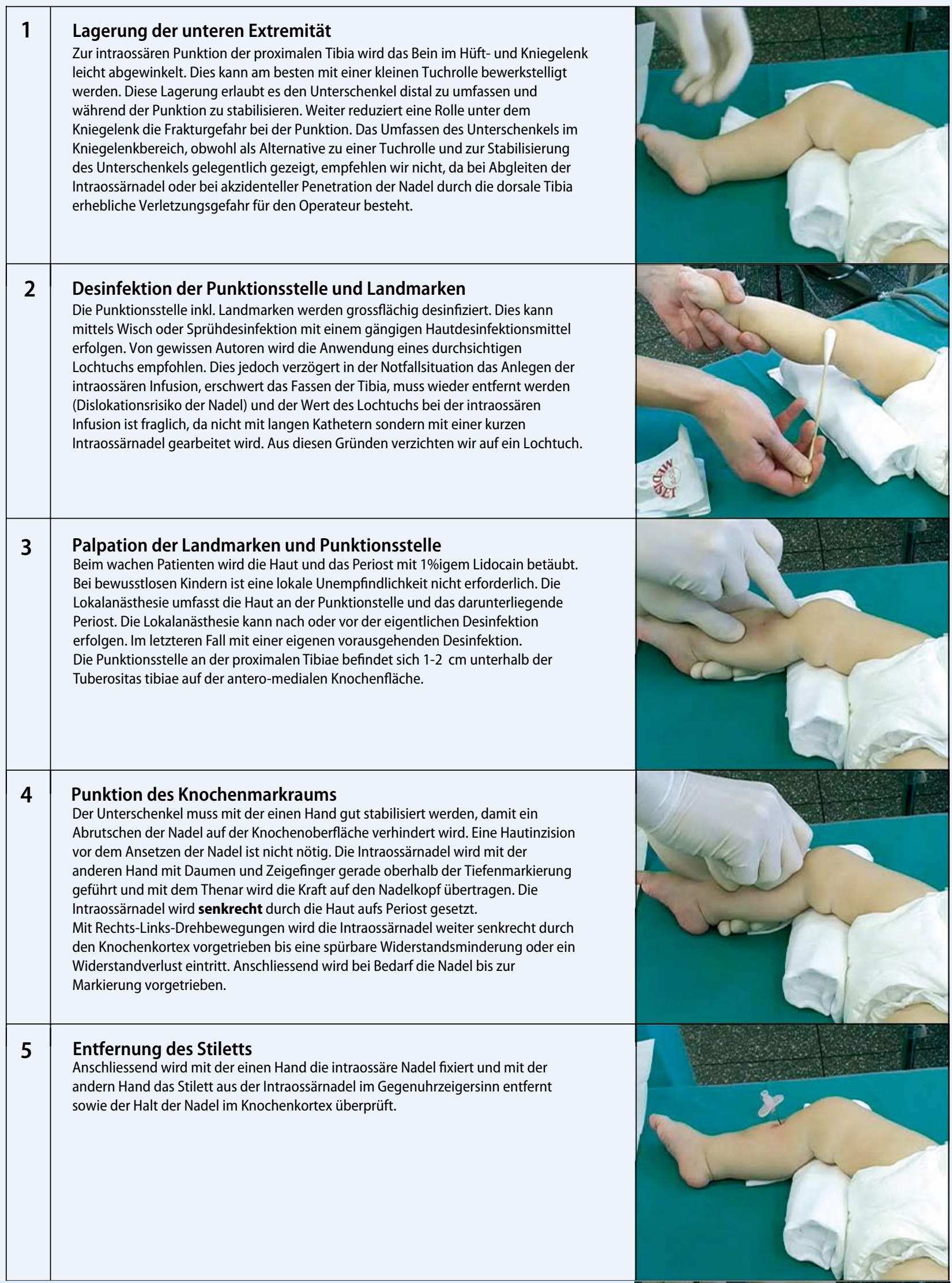

Abb. $9 \Delta$ Technik der intraossären Punktion und Infusion mit der Cook-Intraossärnadel an der proximalen Tibia 


\begin{tabular}{|c|c|}
\hline 6 & $\begin{array}{l}\text { Aspiration von Knochenmark } \\
\text { Mittels einer direkt an die Intraossärnadel angesetzten Spritze kann Knochmark } \\
\text { aspiriert werden. Dass Knochenmark lässt sich dabei, wie auch bei der diagnostischen } \\
\text { Punktion, gelegentlich nur zögerlich aspirieren. Das Aspirat kann für diagnostische } \\
\text { Zwecke verwendet werden. Wichtig: Trotz korrekter Lage kann nicht immer rotes } \\
\text { Knochenmark aspiriert werden. Nach der Aspiration von Knochenmark muss die } \\
\text { Nadel sofort mit physiologischer Kochsalzlösung gespült werden, um eine Okklusion } \\
\text { der Nadel zu vermeiden. Es empfiehlt sich initial } 10 \text { ml Kochsalzlösung forciert zu } \\
\text { injizieren, um im Knochenmark den Weg für die Flüssigkeit etwas zu bahnen. }\end{array}$ \\
\hline 7 & $\begin{array}{l}\text { Konnektion der Infusionsleitung } \\
\text { Ein Infusionsset bestehend aus einer Infusionsleitung, Dreiwegehahn und } \\
\text { einer kurzen Verlängerung wird an die Intraossärnadel angeschlossen. Mittels } \\
\text { des Dreiwegehahns können Medikamente ohne Manipulationen an der } \\
\text { Intraossärnadel appliziert werden. Zur genauen Verabreichung von } \\
\text { Flüssigkeitsmengen kann eine } 20 \mathrm{ml} \text { oder } 50 \mathrm{ml} \text { Spritze an den Dreiwegehahn } \\
\text { angesetzt werden. Wichtig ist zu wissen, dass wache Patienten auf } \\
\text { intramedulläre Druckschwankungen durch Bolus- oder Druckinfusion und } \\
\text { durch Sog mit Schmerzen reagieren können. Die Flüssigkeiten sind } \\
\text { entsprechend langsamer und behutsamer zu verabreichen. Alternativ können } \\
\text { 2-4 ml Lidocain } 1 \% \text { intraossär appliziert werden. }\end{array}$ \\
\hline 8 & $\begin{array}{l}\text { Sicherung der Infusionsleitung } \\
\text { Eine sichere Fixierung der Infusionsleitung ist der beste Schutz der Intraossärnadel } \\
\text { gegen Dislokation, Abbiegen oder gar Abknicken bedingt durch Manipulation oder } \\
\text { akzidentiellen Zug am Infusionssystem. } \\
\text { Das Anschliessen einer kurzen Verlängerung mit Drewegehahn hilft beim Spritzen } \\
\text { Manipulationen an der Intraossärnadel zu vermeiden und das Dislokationsrisiko zu } \\
\text { reduzieren. }\end{array}$ \\
\hline 9 & $\begin{array}{l}\text { Sicherung der Intraossärnadel } \\
\text { Zur Fixierung der Intraossärnadel stehen nur beschränkt Mittel zur Verfügung. } \\
\text { Ein nicht mehr routinemässig mitgelieferter sog. Molnar-Disk, welcher um die } \\
\text { Intraossärnadel angelegt und dann mit Klebeband auf die Haut des Patienten } \\
\text { fixiert werden kann, bietet etwas zusätzliche Stabilität. Gewisse Intraossärnadeln } \\
\text { haben eine Radkappe oder ein Plastikgehäuse, welches bis auf Hautniveau } \\
\text { geschraubt werden kann. Für den Transport des Patienten kann das } \\
\text { entsprechende Bein gegebenenfalls in einer Schiene fixiert werden. }\end{array}$ \\
\hline 10 & $\begin{array}{l}\text { Überwachung der Punktionsstelle } \\
\text { Die Einstichstelle muss sorgfältig überwacht werden, insbesondere auf grössere } \\
\text { Flüssigkeitsaustritte ins Gewebe als Hinweis für eine Fehllage oder Dislokation der } \\
\text { Intraossärnadel. Kleinere Schwellungen aus dem Bohrkanal rund um die Nadel } \\
\text { können gelegentlich vorkommen. Generell vermeiden wir die Verpackung der } \\
\text { Intraossärnadel mit Kompressen und elastischen Binden, da diese die Sicht auf die } \\
\text { Punktionsstellenregion verhindern und kaum zur Stabilität beitragen. }\end{array}$ \\
\hline
\end{tabular}




\section{Leitthema}

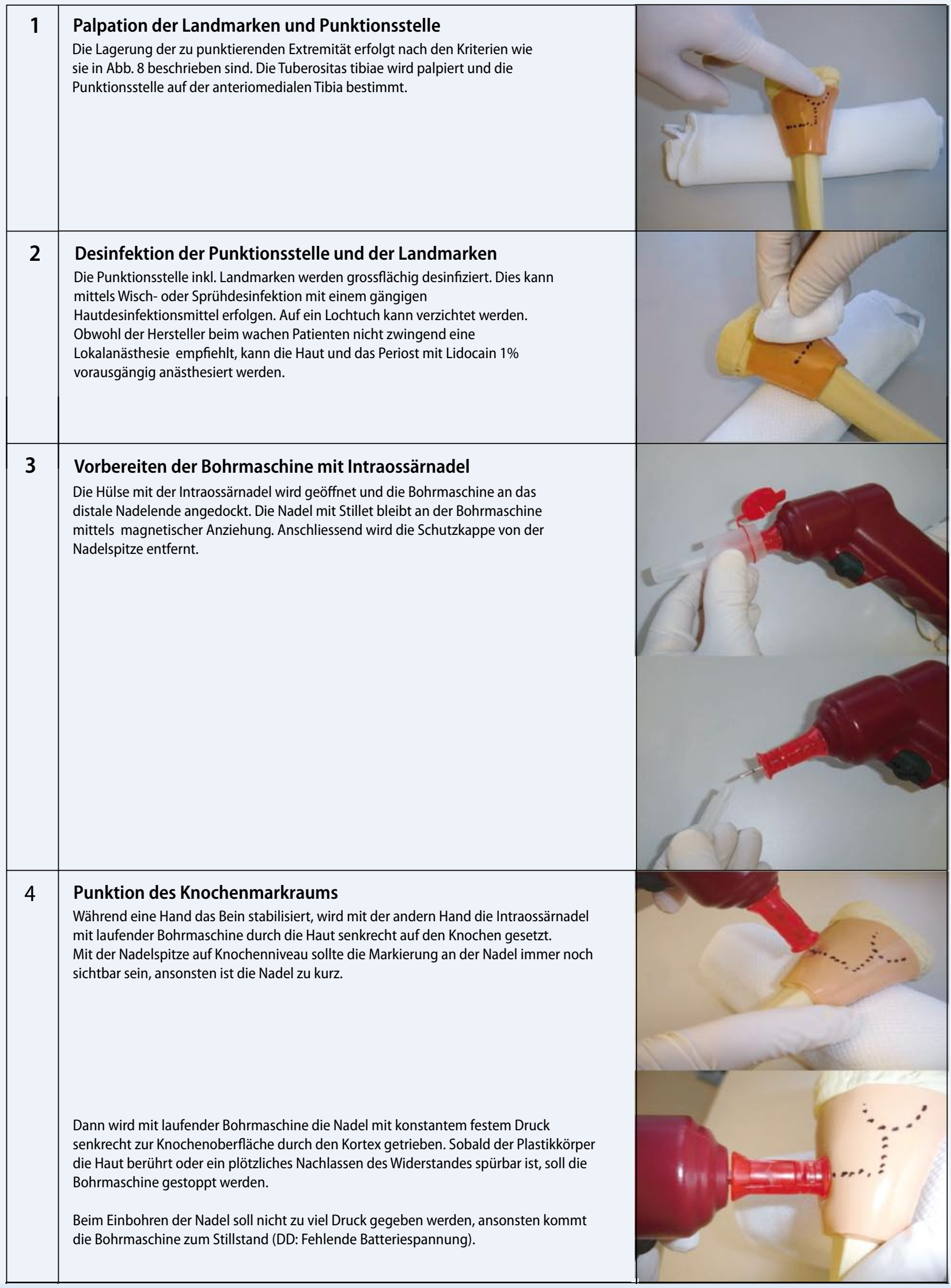

Abb. $10 \Delta$ Technik der intraossären Punktion und Infusion mit dem EZ-IO ${ }^{\text {TM}}$-System an der proximalen Tibia 


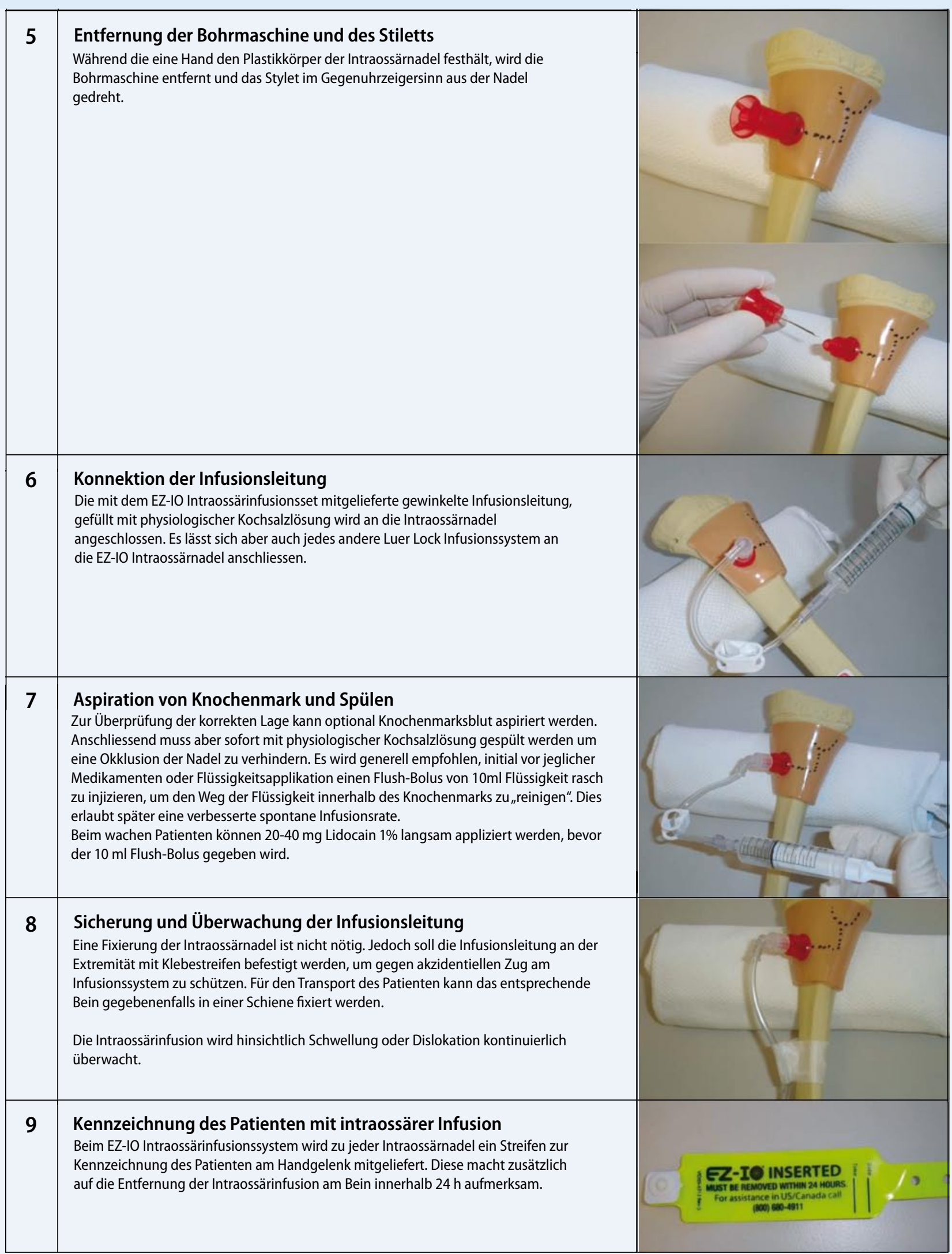




\section{Leitthema}
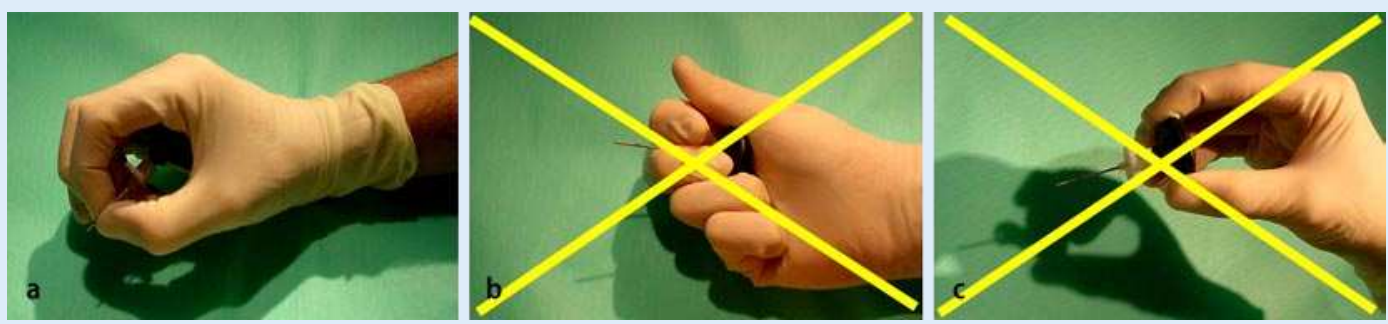

Abb. $11 \Delta$ Korrekte und falsche Handhabung der Cook-Intraossärnadel. Daumen und Zeigefinger fassen und führen die Intraossärnadel an der Tiefenmarkierung, während der Thenar Druck auf den Nadelkopf ausübt (a). Falsche Handhabung der intraossären Nadel (b, c) führt zum Wippen, Abbiegen, Abknicken und Abgleiten der Nadel. Zusätzlich besteht bei falscher Handhabung (b, c) kein Schutz vor zu tiefer Lage, im Extremfall kann es zur dorsalen Penetration der Tibia kommen, wenn die Nadel nicht mit mindestens einem Finger an der Tiefenmarkierung auf Hautniveau gestoppt wird

Für eine erfolgreiche intraossäre Punktion ist nebst der richtigen Lagerung und Fixierung des Unterschenkels [11] und der Wahl der korrekten Punktionsstelle v. a. die richtige Handhabung der Intraossärnadel entscheidend. Der Nadelschaft der Intraossärnadel wird korrekt mit Daumen und Zeigfinger oberhalb der Tiefenmarkierung gefasst und geführt, und mit dem Thenar wird Druck auf den Handgriff (nicht Fingergriff!) appliziert (• Abb. 11). Diese Handhabung der Intraossärnadel erlaubt stabile „Links-Rechts-Drehbewegungen“ mit reduziertem Risiko, vom Knochen abzugleiten, mit der Nadel zu wippen und die Nadel zu verbiegen oder zu knicken.

Der benötigte Druck, um die Nadel einzuführen, ist bei Kleinkindern normalerweise geringer als bei älteren Kindern. Die Einführung einer intraossären Nadel bei älteren Kindern und Erwachsenen kann jedoch mit erheblichem Druckaufwand verbunden sein [9]. Die Distanz zwischen Haut und Knochenmark beträgt selten mehr als einen Zentimeter [52]. Das Führen der Intraossärnadel oberhalb der 1-cm-Tiefenmarkierung verhindert ein zu tiefes Eindringen des Nadelschafts bzw. ein ungebremstes Eindringen mit Penetration der gegenüberliegenden Hinterwand der Tibia.

\begin{tabular}{l} 
Zeichen einer erfolgreichen intraossären \\
Punktion \\
\hline - Widerstandsverlust \\
- Fester Halt der Intraossärnadel ohne Trokar \\
- Aspiration von Knochenmark (beweisend, \\
aber nicht zwingend) \\
- Möglichkeit der Injektion von Flüssigkeit \\
ohne größeren Widerstand und ohne \\
Schwellung \\
Möglichkeit der schwerkraftabhängigen \\
Infusion
\end{tabular}

Die Intraossärnadel soll im 90-Winkel zu Haut- bzw. Knochenoberfläche durch den Knochenkortex gebohrt werden. Andere Autoren wie auch die Herstellerfirma (Cook Critical Care) empfehlen die Nadel schräg und weg von der Diaphyse in den Knochenkortex zu drehen. Bei richtiger Wahl der Punktionsstelle entsprechend den üblichen Kriterien ist dies nicht nötig. Im Gegenteil ist ein schräges Bohren eher nachteilig, da sich das Risiko des Abgleitens, Verbiegens oder gar Abnickens der Nadel massiv erhöht. Zudem ist der Weg durch den Knochenkortex länger und die Tiefenmarkierung ist dann unzuverlässig.

Bei der Verwendung der Cook-Intraossärnadel ist das Manipulieren der Nadel mit sterilen Handschuhen obligat, da der Nadelschaft mit 2 Fingern umfasst wird. Bei anderen Knochenmarknadeln, wie beispielsweise der Baxter-Jamshidi-Intraossärnadel kann die Länge des eindringenden Nadelschafts eingestellt werden und mit den Fingern der Plastikschuh darüber gefasst werden. Ebenso erlauben der BIG oder das EZ-IO ${ }^{\text {mw }}$-Infusionssystem, ohne zusätzliches Anziehen von sterilen Handschuhen die Nadel hygienisch korrekt durch den Knochen zu treiben ( $\bullet$ Abb. 6).

Die Zeichen einer erfolgreichen intraossären Punktion sind [20, 53, 54]:

\section{Plötzlich spürbarer Widerstandsver-}

lust. Der „loss of resistance“ ist ein Hinweis für die Penetration des Knochenkortex $[9,24]$. Beim Neugeborenen ist der Widerstandsverlust eher eine Widerstandsverminderung nach Durchdringen des weichen Knochenkortex. Beim Kleinkind ist der Widerstandsverlust dann deutlich zu spüren und meist auch mit einem deutlich hörbaren Klick verbunden.

\section{Fester Halt der Nadel im Knochenkor-} tex. Ein fester Halt der Intraossärnadel muss auch nach Entfernung des Trokars bestehen bleiben.

Aspiration von rotem Knochenmark. Die Aspiration von rotem Knochenmark zeigt eine korrekte Lage der Intraossärnadel an. Die Aspiration von Knochenmark kann, muss aber trotz korrekter Nadellage nicht positiv sein. Das Knochenmarksaspirat kann zu diagnostischen Zwecken verwendet werden. Der Sog, der dabei notwendig ist, kann erheblich sein, und es braucht auch Geduld [20]. Wichtig: Falls kein rotes Knochenmark aspiriert werden kann, sollte die Nadel nicht sofort entfernt werden, zuerst sollte dann die Möglichkeit der Injektion von Flüssigkeit gestestet werden.

Die Aspiration zur Überprüfung der korrekten Lage wird nicht immer empfohlen. Viele Autoren raten davon ab, da eine erhöhte Gefahr besteht, dass die Intraossärnadel dabei verstopft $[24,55]$. Wird aus einer Intraossärnadel aspiriert, so muss anschließend unmittelbar mit physiologischer Kochsalzlösung oder Ringer-Laktat gespült werden, um eine Obstruktion der Nadel zu verhindern!

Möglichkeit der Flüssigkeitsinjektion. Die Möglichkeit der Injektion von Kochsalzlösung oder Ringer-Laktat ist das sicherste Merkmal zur Überprüfung der korrekten Nadellage [56]. Initial besteht immer ein etwas erhöhter Widerstand, der dann aber abnimmt. Dabei ist die stetige Überprüfung der Weichteile auf Extravasation mit Schwellung wichtig. Ein leichter Flüssigkeitsaustritt durch den Bohrkanal um die Nadel kann vorkommen. 
Schwerkraftabhängige spontane Infusion. Die Infusion sollte, wenn auch langsam, schwerkraftabhängig ohne zusätzlichen Druck frei tropfen. Es wird generell empfohlen, vor jeglicher Medikamenten- oder Flüssigkeitsapplikation einen Flush-Bolus von $10 \mathrm{ml}$ Flüssigkeit rasch zu injizieren, um den Weg der Flüssigkeit innerhalb des Knochenmarks zu „reinigen“. Dies erlaubt später eine verbesserte spontane Infusionsrate, andernfalls ist mit einer verminderten Flussrate oder gar keinem spontanen Fluss zu rechnen.

Nach einem erfolglosen Punktionsversuch oder nach Entfernung einer inkorrekt liegenden oder dislozierten Intraossärnadel darf der selbe Röhrenknochenabschnitt wegen der Gefahr der Extravasation, nicht mehr für weitere Punktionsversuche benutzt werden (absolute Kontraindikation) [22, 57]. Bei der Punktion an der Tibia ist es aber in dieser Situation durchaus gestattet, von der proximalen zur distalen Tibia an der gleichen Extremität zu wechseln und vice versa.

\section{Entfernung der Intraossärnadel}

Die intraossäre Infusion sollte nur solange in situ belassen werden, bis ein periphervenöser oder zentralvenöser Zugang angelegt werden konnte. Generell sollte die Intraossärnadel nach spätestens 12 $(-24)$ h entfernt werden. Für das EZ-IO ${ }^{\mathrm{tm}}$ Intraossärinfusionssystem sind Handgelenksstreifen zur Kennzeichnung des $\mathrm{Pa}$ tienten mit intraossärer Infusion mit der Aufforderung vorhanden, dass diese innerhalb von $24 \mathrm{~h}$ wieder entfernt werden muss. Die Entfernung erfolgt, je nach $\mathrm{Na}$ deltyp, unter leichten Drehbewegungen oder Herausschrauben der Intraossärnadel. Für einige Minuten wird mit einer sterilen Kompresse ein leichter Druck auf die Punktionsstelle ausgeübt, um die Ausbildung eines Hämatoms zu vermeiden. Die Injektionsstelle wird dann für $48 \mathrm{~h}$ steril abgedeckt.

\section{Eine intraossäre Infusion sollte spätestens nach 12-24 h} wieder entfernt werden

Im Knochenkortex kann radiologisch ein kleiner Defekt gefunden werden, welcher sich als kleines Bohrloch, periostale Re- aktion, Aufhellung oder Verdichtung der Kortikalis präsentiert. Diese Defekte können bis zu 6 Monaten nach der Infusion nachweisbar sein, stellen aber keine wirkliche Komplikation dar und müssen nicht therapiert werden $[14,22]$.

\section{Diagnostik aus dem Knochenmarkblut}

Der intraossäre Gefäßzugang ist nicht nur ein wichtiger alternativer Gefäßzugang bei Notfallpatienten zur Verabreichung von Medikamenten und Flüssigkeiten, sondern er erlaubt aspiriertes Knochenmarksblut auch als zuverlässige Quelle für eine initiale Labordiagnostik zu nutzen, wenn keine venöse Blutentnahme möglich ist. Knochenmarkblut aus der intraossär liegenden Nadel zur Bestimmung von Laborparametern vor der Gabe von Flüssigkeiten und Medikamenten kann bedingt wie eine venöse Blutprobe gewertet werden ([58, 59, 6o, 61, 62, 63]; - Tab. 3). Nicht geeignete Parameter sind die Blutenzyme (ALAT, ASAT, LDH, AP) die Thrombozyten-, Leukozyten-, Erythrozytenzahl sowie $\mathrm{pO}_{2}$ - und $\mathrm{SO}_{2}$-Werte. Vorsicht geboten ist beim Kalium, das im Knochenmarkaspirat, am ehesten durch eine sogbedingte Hämolyse, höher als im peripheren Blut sein kann.

Weiter kann das Knochenmarkaspirat auch zum Anlegen von Blutkulturen verwendet werden. Das Knochenmark weist praktisch immer positive Kulturen auf, wenn die peripheren Blutkulturen positiv sind [58].

Verlängerte Versuche, Knochenmark $\mathrm{zu}$ aspirieren, sollten unterlassen werden, um Verzögerungen bei der Verabreichung von lebensrettenden Medikamenten und Flüssigkeiten zu vermeiden und ein Verstopfen der Nadel zu verhindern [59].

Nach Verabreichung von Medikamenten und Infusionslösungen sind die Werte nicht mehr zuverlässig zu verwenden (Verdünnungseffekt), daher sollte Knochenmarkaspirat für Laboruntersuchungen vor der Verabreichung des ersten Medikaments, beziehungsweise der ersten Infusionslösung entnommen und weiterverarbeitet werden.

Des Weiteren können statistische und klinische Unterschiede zwischen venösen und intraossären Werten festgestellt wer-
Tab. 3 Aus dem aspirierten Knochen-

markblut bestimmte Parameter, welche

mit den venösen Blutparametern weit-

gehend übereinstimmen $[58,59,60,61$,

62, 63]

Natrium

Kalium (mit Vorsicht - eher zu hoch wegen

Hämolyse durch Sog)

Chlorid

Frei ionisiertes Kalzium

Magnesium

Anorganisches Phosphat

Laktat

Harnstoff

Harnsäure

Kreatinin

Bilirubin

Glukose

Hämoglobin

Albumin

Gesamtprotein

den, je länger die Dauer der Reanimation ist [63]. Aspiriertes Knochenmark kann aber auch noch nach intraossärer Infusion von Medikamenten und Flüssigkeiten zur genauen und zuverlässigen Bestimmung der Blutgruppe angewendet werden [64].

\section{Blutgasanalyse und Knochenmarkaspirat}

Die intraossären Werte liegen zwischen denen von arteriell und venös entnommenen Proben. Die intraossären Werte können also für die Beurteilung von $\mathrm{pH}$-Wert, $\mathrm{pCO}_{2}$ und $\mathrm{HCO}_{3}$ herangezogen werden [58]. Dies gilt übrigens auch für hypotherme $\mathrm{Pa}$ tienten, Patienten mit Hypoventilation, Hypovolämie oder unter Reanimation [65, $66,67,68]$. Die Blutoxygenierungsparameter $\mathrm{pO}_{2}$ und $\mathrm{SO}_{2}$ sind generell signifikant unterschiedlich zwischen intraossären und peripher arteriellen Werten [59]. Während verlängerten pathophysiologischen Situationen mit prolongierter kardiopulmonaler Reanimation oder andauernder Hypovolämie verändert sich dann aber zunehmend der intraossäre $\mathrm{pH}$ - und $\mathrm{pCO}_{2}$-Wert im Vergleich zu den zentralvenösen Werten $[69,70,71]$. 
Intraossäre Infusion und peripheres Blutbild

Nach intraossärer Infusion von Kochsalz sind im Tierversuch statistisch signifikante Unterschiede in der Zahl der Monozyten, basophilen Granulozyten und Retikulozyten im peripheren Blutbild festgestellt worden $[72,73]$. Daher sollte die Beurteilung des peripheren Blutbilds kurz nach intraossärer Infusion nur mit Vorsicht erfolgen und die Resultate zu einem späteren Zeitpunkt kontrolliert werden.

\section{Medikamentengabe}

Grundsätzlich können alle für die pädiatrische Reanimation und Notfalltherapie benötigten intravenösen Medikamente und Flüssigkeiten nahezu uneingeschränkt intraossär verabreicht werden. Die intraossäre Medikamentendosierung und Flüssigkeitsmenge bleiben gleich wie bei der intravenösen Applikation. Lediglich bei hypertonen Infusionslösungen ist Vorsicht angebracht, da eine erhöhte Infektgefahr besteht. Daher sollten hypertone Lösungen wie Natriumbikarbonat falls möglich verdünnt werden.

Nach Gabe von Medikamenten soll mit 10 ml physiologischer Kochsalzlösung oder Ringer-Laktat nachgespült werden, um den Transport der Medikamente aus dem Knochenmarkraum in die zentrale Zirkulation zu beschleunigen. Besonders wichtig ist dies im Falle eines Herzstillstands [22, 34, 57, 74].

\section{Flussraten}

Die Einlaufgeschwindigkeit intraossär applizierter Lösungen ist geringer als bei der intravenösen Infusion [22]. Die intraossäre Flussrate ist abhängig von verschiedenen Faktoren wie Infusions- und Injektionsdruck, Nadeldicke, Art der applizierten Flüssigkeit, Punktionsstelle bzw. Größe des Knochenmarkraums und Knochenmarkzirkulation. Die erzielten Flussraten von Kristalloiden durch konventionelle manuelle Knochenmarknadeln variieren mit Schwerkraft von $10-17 \mathrm{ml} / \mathrm{min}$ und mit Druck von 41-10o ml/min $[75,76]$.

Bei größeren Kindern und Erwachsenen sind die Flussraten einer intraossären Infusion nicht immer ausreichend für die definitive Therapie von hypovolämen $\mathrm{Zu}$ ständen. Deshalb kann für die Volumensubstitution bei hypovolämen, schockierten Patienten das Anlegen einer bilateralen intraossären Druckinfusion von Nutzen sein $[22,77]$.

Höhere Flussraten können mit modernen intraossären Infusionssystemen erreicht werden, wie dem Pyng F.A.S.T. 1TM IO-Infusionssystem. Damit kann durch Schwerkraftinfusion eine Flussrate von $80 \mathrm{ml} / \mathrm{min}$ erreicht werden, mit Händedruck eine Flussrate von sogar $150 \mathrm{ml} / \mathrm{min}$ [33].

\section{Pharmakokinetik}

Die intraossäre Infusionstechnik ist bezüglich der Effektivität einem intravenösen Zugang gleich zu setzen [11, 12, 78, $79,80,81$. In einigen Studien konnte gezeigt werden, dass während einer kardiopulmonalen Reanimation der intraossäre Zugang mit einem zentralvenösen $\mathrm{Zu}$ gang in der Effektivität vergleichbar ist und einem peripher venösen Zugang sogar überlegen ist $[81,82]$.

\section{Indikation}

Grundsätzlich ist die Indikation für einen intraossären Gefäßzugang für alle vitalen Notfallsituationen bei Patienten $<6$ Jahren gegeben, bei welchen dringend ein Gefäßzugang benötigt wird, jedoch ein Venenzugang nicht oder nur verzögert hergestellt werden kann:

- Bei Kindern mit Herzstillstand [3, 24] oder ausgeprägtem hypovolämischem Schock $[28,54,85]$ soll primär eine intraossäre Infusion angelegt werden, falls nicht schon ein venöser Zugang besteht.

- Bei kritisch kranken Kindern, welche notfallmäßig eine Medikamenten- und Volumengabe zur Wiederherstellung der Vitalfunktion benötigen, ist spätestens nach 3 erfolglosen intravenösen Punktionsversuchen bzw. nach 90-120 s die Indikation für eine intraossäre Infusion gegeben $[24,53,83,86]$.

Beispiele dazu sind: Kreislaufkollaps, Zentralisation bei hypovolämem Schock durch Trauma oder Dehydratation, Krampfanfall mit respiratorischer Insuffizienz, Sta- tus epilepticus, Status asthmaticus, allergische Reaktionen, septischer Schock etc.

Je jünger das Kind, umso schneller wird sich die Indikation für eine intraossäre Injektion im Notfall stellen beziehungsweise ergeben [28]. In Situationen mit offensichtlich erschwerten oder gar unmöglichen Venen- oder Punktionsverhältnissen (Adipositas, Ödeme, Hypothermie, schwere Hypovolämie) oder mit Unmöglichkeit der Ruhigstellung der entsprechenden Punktionsstelle (Epilepsie, unkooperativer Patient, Transporterschütterungen) ist beim vital gefährdeten Patienten die Indikation für eine primär intraossäre Punktion/Infusion gegeben, bevor man aussichtslose Venenpunktionen unternimmt.

\section{Neugeborene}

Bei Neugeborenen sollte im Bedarfsfall eine intraossäre Infusion gelegt werden, falls ein Zugang durch die V. umbilicalis nicht sofort gelingt [87]. Die erfolgreiche Katheterisierung der V. umbilicalis bei Frühgeborenen oder Neugeborenen bedarf großer Erfahrung während der intraossäre Zugang wesentlich einfacher [88] und beim Neugeborenen und Frühgeborenen sehr effektiv ist $[89,90]$. In Langzeituntersuchungen wurden keine Nachteile beim Gebrauch der intraossären $\mathrm{Na}$ deln bei Termin- und Frühgeborenen bezüglich Wachstum der verwendeten Extremität beobachtet [91].

\section{Patienten mit Verbrennungen}

Die Gabe von ausreichend Infusionslösungen und Analgetika ist bei ausgedehnten Verbrennungen ein wichtiger Bestandteil der Erstversorgung. Bei Kindern mit ausgedehnten Verbrennungen kann es schwierig oder gar unmöglich sein, den sofort benötigten Venenzugang zu legen, sodass auch in dieser Situation ein intraossärer Zugang gelegt werden soll [77].

Idealerweise sollte für die intraossäre Punktion eine nicht verbrannte Hautpartie gewählt werden. Falls nicht anders möglich, kann die intraossäre Infusion aber auch durch verbranntes Hautgewebe erfolgen. Die Vorteile einer schnellen Flüssigkeitsgabe überwiegen das relativ kleine Risiko einer Infektion. 
Kinder $>6$ Jahre bzw. Erwachsene

Obwohl es selten ist, dass bei Kindern über 6 Lebensjahren kein venöser Gefäßzugang etabliert werden kann, darf auch hier im Bedarfsfall ein intraossärer Zugang angelegt werden [3]. Dasselbe gilt für erwachsene Patienten, wobei Apparate wie das F.A.S.T.-Infusionssystem, der BIG oder das EZ-IO ${ }^{\text {tw }}$-Intraossärinfusionssystem das Anlegen eines intraossären Zugangs wesentlich erleichtern können [92]. Bei der Verwendung einer manuellen Intraossärnadel empfiehlt es sich bei Kindern $>6$ Lebensjahren und Erwachsenen die distale Tibia als Punktionsort zu wählen.

\section{Kontraindikationen}

\section{Absolute Kontraindikationen}

Wegen der lebensrettenden Bedeutung der intraossären Infusion gibt es grundsätzlich keine generell absoluten Kontraindikationen sondern nur lokoregionale absolute Kontraindikationen, welche den Erfolg der Intraossärinfusion in Frage stellen:

Frakturierte Knochen. Bei Frakturen eines Knochens kommt es zu beträchtlicher Extravasation von intraossär applizierten Medikamenten und Flüssigkeiten ins Gewebe $[20,22,23,57]$. Eine Tibiaplateaufraktur schließt, bedingt durch den zentralen, diaphysären Abfluss jedoch eine Intraossärinfusion an der selben distalen Tibia nicht aus, einmal abgesehen von den Frakturschmerzen bei der Manipulation am wachen Patienten.

\section{Vorausgehende intraossäre Punktions-} versuche. Wurde ein Knochenabschnitt bereits $(<48 \mathrm{~h})$ einmal punktiert und die Nadel wegen Fehlpunktion oder sekundärer Dislokation wieder entfernt, so ist der Knochenkortex nicht mehr intakt. Eine weitere Punktion bzw. Infusion würde zur erheblichen Extravasation führen und die Therapie wäre ineffektiv [93]. Eine Fehlpunktion oder dislozierte Knochenmarknadel an der proximalen Tibia schließt aber, wiederum bedingt durch den zentralen, diaphysären Abfluss des Markblutes eine Intraossärinfusion an der selben distalen Tibia nicht aus.
Gefäßverletzung proximal der intraossären Punktionsstelle. Bei Störungen der regionalen Durchblutung (durch Trauma oder durch vorgängige chirurgische Gefäßexploration) sollte auf die andere Seite oder gegebenenfalls auf die oberen Extremitäten ausgewichen werden [20].

\section{Osteosynthesematerial/voroperierter}

Knochen. Bei Patienten mit vorausgegangenen Operationen oder Osteosynthesematerial im Bereich der Punktionsstelle, sollte keine Intraossärnadel an dieser Stelle angelegt werden.

\section{Relative Kontraindikationen}

In der Literatur ist eine Reihe von relativen Kontraindikationen zu finden (• Tab.4). Diese müssen jedoch im Verhältnis zur Dringlichkeit des Venenzugangs in einer Notfallsituation gesehen werden. Dazu gehören Erkrankungen, die mit einem erhöhten Frakturrisiko einhergehen, wie Osteogenesis imperfecta oder die Osteopetrosis. Ebenso ist ein gesteigertes Osteomyelitisrisko bei Patienten mit Septikämie, Verbrennungen oder Zellulitis zu berücksichtigen. Speziell bei Patienten mit intrakardialen rechts-links Shuntvitien, hochgradiger pulmolarterieller Hypertonie oder schwerer pulmonaler Insuffizienz ist das Risiko der Embolisation von intramedullärem Material, insbesondere bei Druckinfusion zu bedenken. Auch können Erkrankungen wie die akute lymphatische Leukämie, Erythroblastosis, Osteopetrosis zu einem ineffektiven Knochenmark und damit unmöglicher Infusion führen.

Relative Kontraindikationen sind dann relevant, wenn bei schwierigen Venenverhältnissen oder nach mehrfach missglückten Punktionen eine relative Dringlichkeit für einen Venenzugang besteht, wie zum Beispiel bei der intravenösen Narkoseeinleitung eines ASA-IV-Patienten ohne Venenzugang oder bei der Rapid Sequence Induction beim dehydriertem Kind mit Ileus [95]. Letztere Anwendungen werden zurzeit noch stark in Frage gestellt und dürfen noch nicht als generell für die intraossäre Infusion akzeptierte Indikationen angesehen werden $[96,97,98,99,100]$.
Tab. 4 Relative Kontraindikationen für eine intraossäre Punktion und Infusion

Osteogenesis imperfecta $[20,23]$

Osteopetrosis [20]

Erythroblastosis fetalis [11]

Akute lymphatische Leukämie [9]

Intraossäre Nadelplatzierung über verbrannten Arealen $[23,77]$

Intraossäre Nadelplatzierung über Arealen mit Zellulitis [23]

Intrakardialer rechts-links Shunt $[20,94]$

Schwere pulmonale Insuffizienz [95]

Bakteriämie, Septikämie [22]

\section{Komplikationen}

Die Komplikationsrate der intraossären Infusion ist bei korrekter Handhabung gering. Die meisten Berichte über Komplikationen als Folge einer intraossären Infusion sind sehr alt und stammen aus der Zeit, als die Intraossärinfusion noch für routinemäßige Verabreichung von Bluttransfusionen und die Langzeitverabreichung von Medikamenten angewendet wurde. Der heutige selektive Kurzzeiteinsatz in der Notfallmedizin lässt sich daher nur bedingt damit vergleichen.

\section{- Das Risiko der intraossären Infusion steht in keinem Verhältnis zu den Vorteilen beim vital bedrohten Patienten.}

Alle im Zusammenhang mit intraossären Infusionen beschriebenen Todesfälle sind durch Sternalpunktionen und/oder Sternalinfusionen mit Mediastinitis, Hydrothorax oder Verletzung von Herz oder großen Gefäßen beschrieben worden [10, 101, 102] . Die Punktion des Sternums für die intraossäre Infusion ist heute jedoch obsolet in der pädiatrischen Notfallmedizin.

\section{Fehlpunktion und Extravasation}

Die meisten Komplikationen resultieren heute aus inkorrekter Handhabung der Technik und des Materials (• Tab. 5). So ist das Verbiegen der Intraossärnadel der häufigste Grund und die schlecht gewählte Punktionsstelle ein weiterer häufiger Grund für eine erfolglose Punktion [103]. Eine Extravasation von Flüssigkeit aus dem Punktionskanal bei liegender Nadel kommt oft vor und ist in kleinerem Aus- 


\begin{tabular}{l} 
Tab. 5 Komplikationen der intraos- \\
sären Punktion/Infusion \\
\hline $\begin{array}{l}\text { Komplikationen während der Platzierung } \\
\text { der intraossären Nadel: } \\
\text { - Inkorrekte Lage der Einstichstelle } \\
\text { - Abrutschen, Verbiegen und Abbrechen } \\
\text { der intraossären Nadel }\end{array}$ \\
\hline $\begin{array}{l}\text { Schädigung von Knochen (Frakturen), } \\
\text { Knochenmark und Epiphysenfuge }\end{array}$ \\
\hline $\begin{array}{l}\text { Verstopfung der intraossären Nadel, } \\
\text { fehlender Infusionsfluss }\end{array}$ \\
Extravasation und Kompartmentsyndrom \\
\hline $\begin{array}{l}\text { Infektionen (Osteomyelitis, subkutanter } \\
\text { Abszess, Zellulitis, Periostitis) }\end{array}$ \\
\hline Knochenmarkembolien und Fettembolien \\
\hline $\begin{array}{l}\text { Schmerzen durch intraossäre Punktion und } \\
\text { Infusion }\end{array}$ \\
\hline
\end{tabular}

maß um die Einstichstelle normal, unabhängig von Nadeltyp und Druckinfusion [39]. Begünstigend auf die Extravasation sind starke Wippbewegungen beim Eindrehen der Intraossärnadel und Lockerung der Nadel durch häufige Manipulation oder längere Liegedauer [10]. Geschraubte Intraossärnadeln oder mit Apparaturen angelegte intraossäre Gefäßzugänge (• Abb. 5, 6) versprechen diesbezüglich Vorteile. Das seit kurzer Zeit erhältliche EZ- $\mathrm{IO}^{\mathrm{mm}}$-Intraossärinfusionssystem verspricht eine Vereinfachung der Technik, da eine Bohrmaschine den Anwender von Drehbewegungen der Nadel entlastet und er sich ganz auf den Druck und die Bohrrichtung konzentrieren kann. Der Plastikkörper an der Nadel verhindert ein zu tiefes Eindringen der Nadel und bietet gute Stabilität auf der Hautoberfläche.

Unabhängig vom Punktionsmaterial muss immer auf das Auftreten von größeren Schwellungen geachtet werden, um eine Fehllage der Nadel (subkutan, subperiostal, intraartikulär, Durchstechung der hinteren Knochenwand [10]) auszuschließen und Kompartementsyndrome sowie Gewebenekrosen mit anschließender Schwellung und Infektionen (Cave: Adrenalin, Natriumbikarbonat, Kalzium, hypertone Kochsalzlösungen) zu verhindern $[104,105,106]$. Um eine größere Extravasation zu erkennen, sollte die Punktionsstelle nur minimal mit einer Kompresse bedeckt sein [106]. Eine engmaschige Überwachung ist nötig, um ein beginnendes Kompartementsyndrom zu erfassen und gravierende Folgen zu vermeiden.

\section{Frakturen}

Frakturen wurden im Zusammenhang mit sehr kleinen Säuglingen und Neugeborenen und der Anwendung von zu großen Intraossärnadeln und falscher Punktionsstelle berichtet, sind aber sehr selten [107, 108].

\section{Veränderungen des Knochenmarks}

Die intraossäre Verabreichung von Flüssigkeiten und Medikamenten führt $\mathrm{zu}$ vorübergehenden und klinisch nicht relevanten Veränderungen des Knochenmarks (Ödem, entzündliche Infiltration, Zellverarmung, fibrotische und thrombotische Veränderungen bis zu Knochenmarknekrosen; [109, 110]). Die Druckinfusion von isotonen Lösungen hat ebenfalls keine Langzeitauswirkungen [82]. Hypertone Infusionslösungen (Natriumbikarbonat) sollten verdünnt werden [111]. Beinlängenunterschiede nach intraossärer Infusion wurden auch nach Infusion von Natriumbikarbonat nicht gefunden [14, $112,113]$.

\section{Osteomyelitis}

Die Osteomyelitis ist die wichtigste Komplikation. Die Inzidenz ist aber sehr niedrig. In einer von Rosetti et al. an Kindern mit 4270 intraossären Infusionen durchgeführten Untersuchung traten gerade 27 Fälle von Osteomyelitiden auf (o,6\%; [114]). $\mathrm{Zu}$ bedenken ist dabei, dass diese intraossären Infusionen über Tage belassen wurden, während heute empfohlen wird, die intraossäre Infusion sobald wie möglich, spätestens nach 12-24 h wieder zu entfernen. In der neuesten Literatur sind kaum mehr Fälle von Osteomyelitis nach intraossärer Infusion zu finden [95].

Risikofaktoren für eine Osteomyelitis sind unsterile und inkorrekte Punktionstechnik, lang dauernde Infusionen, Infusion von Blutprodukten und hypertonen Lösungen sowie Bakteriämie und Sepsis. Wird die intraossäre Nadel unter notfallmäßigen Bedingungen gelegt, welche eine korrekte sterile Vorgehensweise nicht erlaubten, ist eine Abschirmung mit Antibiotika zu erwägen [20].

\section{Embolisation von Knochenmark und Fett}

Während der intraossären Injektion und Infusion von Medikamenten und Flüssigkeiten besteht die Möglichkeit, dass Fettund Knochenmarkpartikel ins Blut eingeschwemmt werden, welche die Entstehung von Lungenembolien begünstigen können, vor allem wenn intraossäre Infusionen unter Druck verabreicht werden [72, 73]. In einigen Studien konnten regelmäßig histologisch Embolien nachgewiesen werden, in anderen hingegen nicht. Fälle mit klinisch manifesten Lungenembolien insbesondere bei Kindern nach intraossärer Infusion sind bis heute keine beschrieben. Die klinische Relevanz liegt aber darin, dass bei Patienten mit kardialem RechtsLinks-Shunt und schwerer pulmonaler Insuffizienz und/oder pulmonal-arterieller Hypertonie bei der semi-elektiven Indikation einer intraossären Infusion darauf geachtet werden muss [95].

\section{Periostaler oder endostaler Schmerz}

Als nicht unwesentliche Komplikation sind der periostale Schmerz bei der Punktion und der endostale Injektionsschmerz bei der Injektion zu erwähnen. Die intraossäre Infusion sollte beim wachen Patienten nicht ohne Lokalanästhesie des Periosts mit $1 \%$ Lidocain durchgeführt werden. Der periostale Schmerz ist bei Bone Injection Gun (BIG) gering und Bedarf keiner vorausgehenden Lokalanästhesie [31]. Ebenso soll das EZ-IO ${ }^{\mathrm{m}}$-Intraossärinfusionssystem beim Erwachsenen ohne Lokalanästhesie einführbar sein.

Die initiale Injektion der Medikamente verursacht beim wachen Patienten während der ersten Minuten Schmerzen via endostale Schmerzrezeptoren [115]. Langsame Infusionen verursachen praktisch keine Schmerzen [57]. Die endostalen Schmerzen können entweder durch Sog bei der Knochenmarkaspiration, bei der Verabreichung von hypertonen Lösungen oder bei erhöhtem intramedullärem Druck vor allem bei Druckinfusionen entstehen $[14,18,31]$. Bei Bedarf können 2-4 ml Knochenmarkaspirat entnommen und 2-4 ml Lidocain $1 \%$ langsam in das Knochenmark injiziert werden [57]. 


\section{Ausbildung und Material}

Die erfolgreiche, komplikationsarme Anwendung der intraossären Infusionstechnik bedingt einerseits Kenntnisse und Übung im Umgang mit der intraossären Infusionstechnik und andererseits das Vorhandensein der benötigten Ausrüstung dort, wo Kinder behandelt werden.

Jeder ärztliche Mitarbeiter eines medizinischen Systems, welcher potenziell mit Kindern konfrontiert werden kann, muss in diese Technik eingeführt und darin regelmäßig geschult werden. Training in intraossärer Infusionstechnik kann heute an Workshops und notfallmedizinischen Kursen, das nötige Wissen in den entsprechenden Fachzeitschriften und Fachliteratur erworben werden. Für das Erlernen und das Training stehen heute Übungsknochen und Phantome zur Verfügung (• Tab. 6).

Kenntnisse und Übung in intraossärer Infusionstechnik nützen wenig, wenn die benötige Ausrüstung nicht vorhanden ist oder nur schwierig greifbar zur Verfügung steht. Intraossärnadeln oder intraossäre Infusionssets müssen in allen medizinischen Einrichtungen (Praxen, Ambulanzen, Notaufnahmen, Reanimationsausrüstungen, Operationssälen und Intensivstationen) in denen Kinder untersucht, überwacht oder behandelt werden, deutlich gekennzeichnet und ersichtlich gelagert werden. Eine oder 2 Nadelgrößen genügen, und man soll sich dabei auf einen Typ Nadel beschränken, um die Kosten und Logistik in vernünftigen Grenzen zu halten.

\section{Fazit für die Praxis}

Die intraossäre Infusion hat sich in den letzten 20 Jahren zu einem vaskulären Standardzugang in der pädiatrischen und zunehmend auch in der adulten Notfallmedizin entwickelt, wenn nicht innerhalb nützlicher Frist ein venöser Zugang bei lebensbedrohten Patienten gelegt werden kann. Die intraossäre Infusion kann einfach, sicher und schnell mit hoher Erfolgsrate angelegt werden und erlaubt, alle in der Notfallmedizin gebräuchlichen Medikamente fast ohne Einschränkungen zu applizieren. In der lebensbedrohlichen Notfallsituation gibt

Tab. 6 Übungsknochen und Übungsphantome für die intraossäre Infusionstechnik

\begin{tabular}{|c|c|c|}
\hline Megacode Kid & 6 Jahre altes Kind & http://www.global-technologies.net \\
\hline $\begin{array}{l}\text { Life/form }{ }^{\circledast} \text { Intraosseous } \\
\text { Infusion Simulator }\end{array}$ & 6 Monate alter Säugling & http://www.simulaids.com \\
\hline Übungsknochen Tibia rechts & 4 Jahre altes Kind & http://www.synbone.ch \\
\hline Leardal Megacode Baby & 6 Monate alter Säugling & http://www.leardal.de \\
\hline Pediatric ALS Trainer & 6 Monate alter Säugling & http://www.armstrongmedical.com \\
\hline $\begin{array}{l}\text { Nasco's single intraosseous } \\
\text { infusion-leg }\end{array}$ & 6 Monate alter Säugling & http://www.med-mondial.com \\
\hline $\begin{array}{l}\text { Vidacare EZ-IOTM_Übungs- } \\
\text { knochenset }\end{array}$ & $\begin{array}{l}\text { Diverse Modelle (Neuge- } \\
\text { borene bis Erwachsene) }\end{array}$ & http://www.vidacare.com \\
\hline SawBones Worldwide & Diverse Knochenmodelle & http://www.sawbones.com \\
\hline $\begin{array}{l}\text { Adult Sternal Intraosseous } \\
\text { Infusion Simulator }\end{array}$ & $\begin{array}{l}\text { Oberer Thorax eines } \\
\text { Erwachsenen }\end{array}$ & http://www.drmass.com \\
\hline
\end{tabular}

es, abgesehen von lokalen Gründen, keine absoluten Kontraindikation für eine intraossäre Infusion. Das Komplikationsrisiko ist klein und rechtfertigt nicht, auf die Vorteile der Intraossärinfusion bei gegebener Indikation zu verzichten. Misslingen der intraossären Infusion ist meist bedingt durch inkorrekte Handhabung von manuellen Intraossärnadeln. Geräte wie die Bohrmaschinen unterstützte Insertion von Knochenmarknadeln mit Distanzbolzen mögen in Zukunft auch bei Kindern die Erfolgsrate erhöhen. Kenntnisse und Übung in der intraossären Infusionstechnik gehören zum Bestandteil der Ausbildung und des kontinuierlichen Trainings jedes Notfallmediziners, Notarztes, Pädiaters, Anästhesisten und Intensivmediziners.

\section{Korrespondierender Autor \\ PD Dr. M. Weiss}

Anästhesieabteilung

Universitäts-Kinderkliniken Zürich

Steinwiesstrasse 75, 8032 Zürich, Schweiz

markus.weiss@kispi.unizh.ch

Copyrights Das Videomaterial ist in anderer Aufmachung bereits Bestandteil einer Lehr-CD der Universität Bern, Schweiz und online via Inselspital, Bern und via KATZ'07 (http://www.kindernarkose.ch) der Öffentlichkeit zugänglich. Die Copyrights des Videomaterials gehören der Anästhesieabteilung, Universitäts-Kinderkliniken Zürich.

Interessenkonflikt. Die Autoren versichern, dass keine Verbindungen mit einer Firma, deren Produkt im Artikel aufgeführt ist, oder einer Firma, die ein Konkurrenzprodukt vertreibt, besteht.

\section{plus Video}

Abbildungen mit diesem Symbol enthalten zusätzliches Videomaterial. Bitte klicken Sie zum Abspielen des Videos auf die jeweilige Abbildung in der PDF-Datei des Beitrags. Diese finden Sie auf www.NotfallundRettungsmedizin.de unter "Inhalte/Archiv“.

\section{Literatur}

1. Rossetti V, Thompson BM, Aprahamian C (1984) Difficulty and delay in intravascular access in pediatric arrest. Ann Emerg Med 13: 406

2. American Heart Association (2005) Part 12. Pediatric Advanced Life Support. Circulation 112: 167187; American Heart Association Guidelines for Cardiopulmonary Resuscitation and Emergency Cardiovascular Care (2005) Part 12. Pediatric Advanced Life Support. Circulation 112: IV167-187

3. The International Liaison Committee on Resuscitation (ILCOR) (2005) Consensus Science with treatment recommendations for pediatric and neonatal patients: Pediatric Basic and Advanced Life Support. Pediatrics 117: e955-977

4. European Resuscitation Council Guidelines for Resuscitation (2005) Section 6. Paediatric life support. Resuscitation 67S1: 97-133; Section 4. Adult advanced life support. Resuscitation 67S1: 39-86

5. Drinker CK, Drinker KR (1916) A method for maintaining an artificial circulation through the tibia of the dog, with a demostration of the vasomotor control of the marrow vessels. Am J Physiol XL: 514-521

6. Drinker CK, Drinker KR (1922) The circulation in the mammalian bone marrow. Am J Physiol 62: 1-92

7. Doan CA (1922) The circulation of the bone marrow. Contrib Embryol 67: 27-47

8. Tocantins LM, O'Neill JF, Price AH (1941) Infusions of blood and other fluids via the bone marrow in traumatic shock and other forms of peripheral circulatory failure. Ann Surg 114: 1085-1092

9. Tocantins LM, Price AH, O'Neill JF (1943) Infusions via the bone marrow in children. Pennsylvania Med J 46: 1267-1273

10. Tocantins LM, O'Neill JF (1945) Complications of intra-osseous therapy. Ann Surg 122: 266-277

11. Tocantins LM, O'Neill JF, Jones HW (1941) Infusions of blood and other fluids via the bone marrow application in pediatrics. JAMA 117: 1229-1234 
12. Tocantins LM, O'Neill JF (1940) Infusion of blood and other fluids into circulation via the bone marrow. Proc Soc Exp Biol Med 45: 782-783

13. Tocantins LM, O'Neill JF (1941) Infusions of blood and other fluids into general circulation via bone marrow. Technique and results. Surg Gynec and Obst 73: 281-287

14. Heinild S, Sondergaard T, Tudvad F (1947) Bone marrow infusion in childhood. Experiences from a thousand infusions. J Pediatr 30: 400-412

15. Papper EM, Rovenstine EA (1942) Utility of marrow cavity of sternum for parenteral fluid therapy. War Med 2: 277-283

16. Turkel H (1983) Intraosseous infusion (Letter). Am J Dis Child 137: 706

17. Orlowski JP (1984) My kingdom for an intravenous line (Editorial). Am J Dis Child 138: 803

18. Tarrow AB, Turkel H, Thompson S (1952) Infusions via the bone marrow and biopsy of the bone and bone marrow. Anesthesiology 13: 501-509

19. Henning N(1945) Intrasternal Infusion.JAMA 128:240

20. Orlowski JP (1994) Emergency alternatives to intravenous access. Intraosseous, intratracheal, sublingual and other-site drug administration. Ped Crit Care 41: 1183-1199

21. Gimson JD (1944) Bone-marrow transfusion in infants and children. BMJ 1: 748-749

22. Spivey WH (1987) Intraosseous infusions. J Pediatr 111:639-643

23. Hodge D (1985) Intraosseous infusions: a review. Pediatr Emerg Care 1: 215-218

24. Seigler RS, Tecklenburg FW, Shealy R (1989) Prehospital intraosseous infusion by emergency medical services personnel: a prospective study. Pediatrics 84: 173-177

25. Iserson KV, Criss E (1986) Intraosseous infusions: a usable technique. Am J Emerg Med 4: 540-542

26. Iserson KV (1989) Intraosseous infusions in adults. J Emerg Med 7: 587-591

27. Schoffstall JM, Spivey WH, Davidheiser S (1989) Intraosseous crystalloid and blood infusion in a swine model. J Trauma 29: 384-387

28. Manley L, Haley K, Dick M (1988) Intraosseous infusion: rapid vascular access for critically ill or injured infants and children. J Emerg Nursing 14: 63-69

29. Reisman HA, Tainsky IA (1944) Bone marrow as alternative route for transfusion in pediatrics. Am J Dis Child 68: 253-256

30. Elston JT, Jaynes RV, Kaump DH (1947) Intraosseous infusion in infants. Am J Clin Pathol 17: 143-150

31. Waisman M, Waisman D (1997) Bone marrow infusion in adults. J Trauma 42: 288-293

32. Warren DW, Kissoon N, Sommerauer JF, Rieder M (1993) Comparison of fluid infusion rates among pe ripheral intravenous and humerus, femur, malleolus and tibial intraosseous sites in normovolemic and hypovolemic piglets. Ann Emerg Med 22: 183-186

33. Macnab A, Christenson J, Findlay J et al. (2000) A new system for sternal intraosseous infusion in adults. Prehosp Emerg Care 1: 173-1777

34. Seefelder C, Ahnefeld FW, Stannigel H (1992) Die Stellung der intraossäre Injektion und Infusion bei pädiatrischen Notfällen. Eine Literaturübersicht. Notarzt 8: 175-183

35. Wagner M, Mc Cabe J (1987) A comparison of four techniques to establish intraosseous infusion. Ann Emerg Med 16: 509

36. Daga SR, Gosavi DV, Verma B (1999) Intraosseous ac cess using butterfly needle. Trop Doct 29: 142-144

37. Lake W, Emmerson AJ (2003) Use of butterfly as an intraosseous needle in an oedematous preterm infant. Arch Dis Child Fetal Neonatal 88: 409

38. Ellemunter $H$, Burkhart S (1999) Intraosseous lines in preterm and full term neonates. Arch Dis Child Fetal Neonatal 80: 74-75
39. LaSpada J, Kissoon N (1995) Extravasation rates and complications of intraosseous needles during gravity and pressure infusion. Crit Care Med 23: 2023-2028

40. Glaeser PW, Losek JD (1988) Intraosseous needles. New and improved. Pediatr Emerg Care 4: 135-136

41. Miller DD, Guimond G (2005) Feasibility of sternal intraosseous access by emergency medical technical students. Prehosp Emerg Care 9: 73-78

42. DeBoer S, Seaver M, Morissette C (2005) Intraosseous infusion: not just for kids anymore. Emerg Med Serv 34: 54-63

43. Koschel MJ (2005) Sternal intraosseous infusions: emergency vascular access in adults. Am J Nurs 105: 66-68

44. Miller L, Kramer GC (2005) Rescue access made easy. JEMS 30: 8-18

45. Macnab A, Christenson J, Findlay J et al. (2000) A new system for sternal intraosseous infusion in adults. Prehosp Emerg Care 1: 173-177

46. Calkins D, Fitzgerald G (2000) Intraosseous infusion devices. a comparison for potential use in special operations. J Trauma 48: 1068-1074

47. Vardi A, Berkenstadt H (2004) Intraosseous vascular access in the treatment of chemical warfare casualties assessed by advanced simulation: Proposed alteration of treatment protocol. Anesth Analg 98: $1753-1758$

48. Seibert FJ, Schatz B (2001) Bone Injection Gun (B.I.G.) im Test. Notarzt 17: 152-154

49. Heightman AJ (2005) The rebirth of adult IO. JEMS 30:4-7

50. Davidoff J, Fowler R, Gordon D et al. (2005) Clinical evaluation of a novel intraosseous device for adults: prospective, 250-patient, multi-center trial. JEMS 30: 20-23

51. Frascone RJ, Jensen J, Salzman J, Kaye K (2006) EZIO: a field trial. NAEMSP Annual Meeting, January 19-21, 2006, Tucson, AZ

52. Holder M (1991) Intraossäre Injektion und Infusion im Kindesalter. Notfallmedizin 17: 648-652

53. Helm M, Breschinski W, Lampl L (1996) Die intraossäre Punktion in der präklinischen Notfallmedizin. Praktische Erfahrungen aus dem Luftrettungsdienst. Anästhesist 45: 1196-1202

54. Berg RA (1984) Emergency infusion of catecholamines into bone marrow. Am J Dis Child 138: 810-811

55. Simmons CM, Johnson NE, Perkin RM, Stralen D van (1994) Intraosseous extravasation complication reports. Ann Emerg Med 23: 363-366

56. Peck KR, Altieri M (1988) Intraosseous infusions: an old technique with modern applications. Pediatr Nursing 14: 296-298

57. Mofenson HC (1988) Guidelines for intraosseous infusions. J Emerg Med 6:143-146

58. Orlowski JP, Porembka DT, Gallagher JM (1989) The bone marrow as a source of laboratory studies. Ann Emerg Med 18: 1348-1351

59. Grisham J, Hastings C (1991) Bone marrow aspirate as an accessible and reliable source for critical laboratory studies. Ann Emerg Med 20: 1121-1124

60. Ummenhofer W, Frei FJ, Urwyler A (1994) Are laboratory values in bone marrow aspirate predictable for venous blood in pediatric patients? Resuscitation 27: 123-128

61. Hurren JS (2000) Can blood taken from intraosseous cannulations be used for blood analysis? Burns 26: 727-730

62. Unger H, Spivey WH (1986) Comparison of intraosseous and intravenous $\mathrm{CBC}$ and Astra 8 in swine (Abstract). Ann Emerg Med 15: 647

63. Johnson L, Kissoon N, Fiallos M et al. (1999) Use of intraosseous blood to assess blood chemistries and hemoglobin during cardiopulmonary resuscitation with drug infusions. Crit Care Med 27: 1147-1152
64. Brickman KR (1992) Typing and screening of blood fromintraosseousaccess.AnnEmergMed21:414-417

65. Kissoon N, Rosenberg H, Gloor J (1993) Comparison of acid-base status of blood obtained from the intraosseous and central venous site during steady and low flow states. Crit Care Med 21: 1765-1769

66. Kissoon N, Peterson R, Murphy S (1994) Comparison of $\mathrm{pH}$ and carbon dioxide tension values of central venous and intraosseous blood during changes in cardiac output. Crit Care Med 22: 1010-1015

67. Brickman K, Rega P (1987) A comparative study of intraosseous, intravenous and intraarterial $\mathrm{pH}$ changes during hypoventilation in dogs. Ann Emerg Med 16: 510

68. VoelckelWG (2000) Intraosseous blood gases during hypothermia. Correlation with arterial, mixed venous and sagittal sinus blood. Crit Care Med 28: 2915

69. Kissoon N, Idris A, Wenzel V (1997) Intraosseous and central venous blood acid-base relationship during cardiopulmonary resuscitation. Pediatr Emerg Care 13: 250-253

70. Kiaer T, Gronlund J (1990) Effects of variation in systemic blood pressure on intraosseous pressure, PO2, and PCO2. J Orthop Res 8: 618-622

71. Abdelmoneim T, Kissoon N (1999) Acid-base status of blood from intraosseous and mixed venous sites during prolonged cardiopulmonary resuscitation and drug infusions. Crit Care Med 27: 1923

72. Ros SP (1991) Effect of intraosseous saline infusion on hematologic parameters. Ann Emerg Med 20: 243

73. Joffe M (1990) Blasts in peripheral blood with intraosseous infusion. Pediatr Emerg Care 6: 106

74. Spivey WH, Lathers CM, Malone D (1985) Comparison of intraosseous, central and peripheral routes of administration of sodium bicarbonate during CPR in pigs. Ann Emerg Med 14: 1135-1140

75. Schoffstall JM, Spivey WH, Davidheiser S (1989) Intraosseous crystalloid and blood infusion in a swine model. J Trauma 29: 384-387

76. Shoor PM, Berryhill RE, Benumof JL (1979) Intraosseous infusion: pressure-flow relationship and pharmakinetics. JTrauma 19: 772-774

77. Goldstein B, Doody D, Briggs S (1990) Emergency intraosseous infusion in severly burned children. Pediatr Emerg Care 6: 195-197

78. Orlowski JP, Porembka DT, Gallagher JM et al. (1990) Comparison study of intraosseous, central intravenous, and peripheral intravenous infusions of emergency drugs. Am J Dis Child 144: 112-117

79. Warren DW, Kissoon N, Mattar A (1994) Pharmacokinetics from multiple intraosseous and peripheral intravenous site injections in normovolemic and hypovolemic pigs. Crit Care Med 22: 838-843

80. Tocantins LM (1940) Rapid absorption of substances injected into the bone marrow. Proc Soc Exp Biol Med 45: 292-296

81. Cameron JL, Fontanarrosa PB, Passalaqua AM (1989) A comparative study of peripheral to central circulation delivery times between intraosseous and intravenous injection using a radionuclide technique in normovolemic and hypovolemic canines. J Emerg Med 7: 123-127

82. Neufeld JD (1993) Comparison of intraosseous, central, and peripheral routes of crystalloid infusion for resuscitation of hemorrhagic shock in a swine model. J Trauma 34: 422-428

Das komplette Literaturverzeichnis ...

... finden Sie in der elektronischen Version dieses Beitrags unter www.NotfallundRettungsmedizin.de 